\title{
Probing the origin of low-frequency radio emission in PG quasars with the uGMRT - I
}

\author{
Silpa S., ${ }^{1 \star}$ P. Kharb, ${ }^{1}$ L. C. Ho, ${ }^{2,3}$ C. H. Ishwara-Chandra ${ }^{1}$ M. E. Jarvis, ${ }^{4,5,6}$ C. Harrison ${ }^{7}$ \\ ${ }^{1}$ National Centre for Radio Astrophysics - Tata Institute of Fundamental Research, S. P. Pune University Campus, Ganeshkhind, Pune 411007, India \\ ${ }^{2}$ Kavli Institute for Astronomy and Astrophysics, Peking University, Beijing 100871, China \\ ${ }^{3}$ Department of Astronomy, School of Physics, Peking University, Beijing 100871, China \\ ${ }^{4}$ Max-Planck Institut für Astrophysik, Karl-Schwarzschild-Str. 1, 85748 Garching, Germany \\ ${ }^{5}$ European Southern Observatory, Karl-Schwarzschild-Str. 2, 85748 Garching, Germany \\ ${ }^{6}$ Ludwig Maximilian Universität, Professor-Huber-Platz 2, 80539 Munich, Germany \\ ${ }^{7}$ School of Mathematics, Statistics and Physics, Newcastle University, Newcastle upon Tyne, NE1 7RU, UK
}

22 December 2020

\begin{abstract}
We present the results from $685 \mathrm{MHz}$ observations with the upgraded Giant Metrewave Radio Telescope (uGMRT) of 22 quasars belonging to the Palomar-Green (PG) quasar sample. Only four sources reveal extended radio structures on $\sim 10-30 \mathrm{kpc}$ scales, while the rest are largely a combination of a radio core unresolved at the uGMRT resolution of $\sim 3-5^{\prime \prime}$, surrounded by diffuse emission on few $\mathrm{kpc}$ to $\sim 10 \mathrm{kpc}$ scales. A few sources reveal signatures of barely resolved jets and lobes in their spectral index images that are created using the uGMRT 685 $\mathrm{MHz}$ data and similar resolution GHz-frequency data from the Very Large Array. On the basis of their position on the radio-IR correlation as well as the spectral index images, we find that the radio emission in the two radio-loud (RL) quasars and nearly one-third of the radio-quiet (RQ) quasars is active galactic nucleus (AGN) dominated whereas the remaining sources appear to have significant contributions from stellar-related processes along with the AGN. While the two RL sources exhibit inverted spectral index in their cores, the RQ sources exhibit a range of spectral indices varying from flat to steep $\left(-0.1 \gtrsim \alpha_{R} \gtrsim-1.1\right)$ indicating the presence of unresolved jets/lobes or winds. Except for a significant correlation between the $685 \mathrm{MHz}$ radio luminosity and the Eddington ratio, we do not find strong correlations between other $685 \mathrm{MHz}$ radio properties and black hole (BH) properties in the RQ PG sources. This lack of correlations could be explained by the contribution of stellar-related emission, or radio emission from previous AGN activity episodes which may not be related to the current $\mathrm{BH}$ activity state.
\end{abstract}

Key words: techniques: interferometric -- quasars: general -- radio continuum: general

\section{INTRODUCTION}

Active galactic nuclei (AGN) are the energetic centres of galaxies that are believed to be powered by the gravitational energy released from accretion of matter on to supermassive black holes $\left(10^{5}-\right.$ $10^{10} M_{\odot}$; see review by Rees 1984). Bipolar outflows are launched perpendicular to the black hole-accretion disc systems. It is unclear why only $10-20 \%$ of AGN launch powerful radio outflows (e.g., Kellermann et al. 1989; Urry 2003) that extend to hundreds of kiloparsecs or megaparsecs (the so called 'radio-loud', RL AGN), while the vast majority of AGN launch small (parsec-scale) or weak outflows that are typically $\lesssim 10 \mathrm{kpc}$ in extent and many a times bubble-/wind-like (the 'radio-quiet', RQ AGN). The radio-loudness parameter (R) given by Kellermann et al. (1989), which is the ratio

\footnotetext{
^ E-mail: silpa@ncra.tifr.res.in
}

of radio flux density at $5 \mathrm{GHz}$ to optical flux density in the optical B-band, is often used to separate these two classes: RL AGN have $\mathrm{R}>10$ and RQ AGN have $\mathrm{R} \leq 10$. The influence of host galaxy on the type of outflows observed is also unclear since RL AGN are mostly hosted by elliptical galaxies whereas RQ AGN reside in both spiral and elliptical galaxies. Differences in black hole (BH) masses, spins and accretion rates have often been suggested to explain this RL-RQ divide (Blandford \& Rees 1974; Wilson \& Colbert 1995; Tchekhovskoy et al. 2010). It is proposed in literature that highmass BHs reside in RL sources whereas low-mass BHs reside in RQ sources (Dunlop et al. 2003; McLure \& Jarvis 2004). Also, radio-loudness decreases with increasing Eddington ratio (Ho 2002; Sikora et al. 2007). However, while some authors suggest that the radio-loudness distribution is bimodal (Kellermann et al. 1989), or at least not unimodal (Kratzer \& Richards 2015), others suggest that the distribution is continuous (White et al. 2000; La Franca et al. 2010). This RL-RQ dichotomy can be explained by understanding 
the different physical mechanisms contributing to radio emission in these two classes of AGN, namely RL and RQ.

In RL AGN, accretion on to BHs produces collimated relativistic jets that emit synchrotron radiation. The jets are generally accepted to be produced by the electromagnetic extraction of rotational energy from spinning BHs (Blandford \& Znajek 1977). However, the origin of radio emission in RQ AGN is poorly understood (Panessa et al. 2019). Possible origins include: (1) thermal free-free emission from $\mathrm{H}$ II regions around young, massive stars like $\mathrm{O}$ - and B-type stars (Terzian 1965); (2) non-thermal synchrotron emission associated with starburst superwinds (Condon et al. 2013; Kellermann et al. 2016); (3) coronal emission due to magnetic heating of the corona, presumably located above the accretion disc (Laor \& Behar 2008; Raginski \& Laor 2016); (4) optically thin free-free emission from a slow, dense accretion disc wind (Blundell \& Kuncic 2007) or from the torus (Carilli et al. 2019) (however see Ho 1999; Lal \& Ho 2010); (5) compact or low-powered radio jets on $<\sim$ kpc scales (Falcke et al. 2000; Jarvis et al. 2019; Kharb et al. 2019); (6) a combination of both AGN and starburst-driven winds (Cecil et al. 2001; Irwin \& Saikia 2003; Hota \& Saikia 2006); (7) AGN-driven winds, which can be launched and accelerated by the following mechanisms: (i) thermal-driving (Begelman et al. 1983; Woods et al. 1996; Mizumoto et al. 2019); (ii) radiative-driving (Proga \& Kallman 2004; Zakamska \& Greene 2014; Nims et al. 2015); (iii) magnetic-driving (Blandford \& Payne 1982; Konigl \& Kartje 1994; Everett 2005; Fukumura et al. 2010).

In this paper, we present a $685 \mathrm{MHz}$ study of 22 Palomar-Green (PG) quasars using the upgraded Giant Metrewave Radio Telescope (uGMRT) to address the question of the origin of radio emission in RQ AGN. Our aim with the uGMRT was to observe and understand the origin of low-frequency diffuse and extended emission in the PG quasars at resolutions that are intermediate between the previous Very large Array (VLA) A and D-array $5 \mathrm{GHz}$ observations $\left(0.5^{\prime \prime}\right.$ and $18^{\prime \prime}$ respectively; Kellermann et al. 1989, 1994). The 685 $\mathrm{MHz}$ observations is the best compromise between resolution and sensitivity with the uGMRT. These observations provide an angular resolution of $\sim 4^{\prime \prime}$ which corresponds to spatial extents of few kpc to tens of kpc in the PG sources. As these sources have extensive $\mathrm{GHz}$ frequency radio data available from the VLA, we aim to obtain information about spectral indices and determine the spectral ages of outflows using uGMRT and VLA data. Our goal is to also constrain the AGN duty cycle by detecting emission from multiple activity episodes of the AGN that is presumably steep spectrum and therefore bright at low frequencies. We have also obtained polarimetric data with the uGMRT at $685 \mathrm{MHz}$, which we will present in a forthcoming paper (Silpa et al. 2021, in preparation).

The paper is organized in the following manner: Section 2 describes the PG sample and Section 3 describes the uGMRT data reduction and analysis. We present our results in Section 4 and conclusions in Section 5. Throughout this paper, we have assumed $\Lambda$ cold dark matter cosmology with $H_{0}=73 \mathrm{~km} \mathrm{~s}^{-1} \mathrm{Mpc}^{-1}, \Omega_{m}=$ 0.27 and $\Omega_{v}=0.73$. The spectral index $\alpha_{R}$ is defined such that flux density at frequency $v, S_{v} \propto v^{\alpha_{R}}$.

\section{THE SAMPLE}

The PG catalogue of $\sim 1800$ UV-excess (i.e., $\mathrm{U}-\mathrm{B}<-0.44$ ) objects is derived from a survey covering an area of $\sim 10,714 \mathrm{deg}^{2}$ in absolute galactic latitudes above $30^{\circ}$, using 266 double $\mathrm{U}$ and $\mathrm{B}$ exposures of the Palomar 18 inch Schmidt telescope (Green et al. 1986). The Palomar Bright Quasar Survey (BQS), which is a subset of the larger PG survey, included only those objects which fulfilled the (1) morphological criteria: dominant star-like appearance; (2) spectroscopic criteria: presence of broad emission lines. The BQS sample consisted of 114 objects, which included 92 Quasars with $M_{B}<-23$ and 22 Seyferts or low-luminosity quasars with $M_{B}>$ -23 . The PG quasar sample, which is our sample of interest here, is composed of objects from BQS with $z<0.5$. These are 87 sources comprising of quasars and Seyfert type 1 galaxies (Boroson \& Green 1992). Nearly $80 \%$ of this sample is RQ, while $\sim 20 \%$ is RL.

The PG quasar sample is one of the most well-studied samples of low-redshift AGN. The wealth of data that exists for this sample includes: (a) accurate $\mathrm{BH}$ masses measured from reverberation mapping (Kaspi et al. 2000) and single-epoch spectroscopy data (Vestergaard \& Peterson 2006); (b) host galaxy morphologies and galaxy bulge/disk decompositions from HST imaging data (Kim et al. 2008, 2017); (c) complete broad-band spectral energy distributions (SEDs) and accurate bolometric luminosities from data covering radio to hard X-rays (Shang et al. 2011); (d) observations of dust (Petric et al. 2015; Shangguan et al. 2018) and gas (Evans et al. 2006; Shangguan et al. 2020) properties, enabling examination of the interstellar medium of the host galaxies; and (e) IR data determining torus properties (Zhuang et al. 2018) and star formation rates (SFRs) (Shi et al. 2014, Xie et al. 2020, submitted). Despite the immense wealth of data, there is lack of high-resolution, high-sensitivity low-frequency radio data for this sample.

In this paper, we present results from $685 \mathrm{MHz}$ observations of 22 (out of 87) PG quasars with the uGMRT. This sub-sample was a result of the limited approved time to carry out an initial pilot study with the uGMRT. We therefore chose targets that had recently been observed with the Atacama Large Millimeter/submillimeter Array (ALMA) by Shangguan et al. (2020), with the aim of looking at the low-frequency radio emission in tandem with $\mathrm{CO}(2-1)$ molecular emission. The results of this work will be presented in a future paper.

\section{OBSERVATIONS AND DATA REDUCTION}

\section{1 uGMRT observations}

The uGMRT observations of the PG quasar sample at $685 \mathrm{MHz}$ (Band 4: 550-850 MHz) were carried out on 2018 November 23 and 2018 December 7 (project code 35_042). The uGMRT has a seamless frequency coverage from 50 to $1500 \mathrm{MHz}$ and instantaneous bandwidth of $400 \mathrm{MHz}$. The data sets obtained on both dates were individually reduced and analysed using the standard procedures in CASA $^{1}$. 27 antennas with $400 \mathrm{MHz}$ bandwidth and 4096 channels were used; 3 antennas were not working during the observations. The details of these observations are given in Table 1. Each source was observed for at least two scans of $\sim 20$ min each for better $u v$ coverage. The flux density calibrators (3C 48 and 3C 286) were observed for $5 \mathrm{~min}$ at the beginning and end of the observations and the phase calibrators (listed in Table 1) were observed for $5 \mathrm{~min}$ once every $\sim 20 \mathrm{~min}$ of the on-source time.

\subsection{Data reduction and imaging}

The LTA data files produced by GMRT were first converted into FITS format by LISTSCAN and GVFITS utilities. The usable bandwidth of uGMRT Band 4 data is $560-810 \mathrm{MHz}$ only, since the sen-

1 Common Astronomy Software Applications; Shaw et al. (2007) 
Table 1. uGMRT observation details and VLA archival data details of PG quasars.

\begin{tabular}{|c|c|c|c|c|c|c|c|c|c|}
\hline Quasar & $\begin{array}{c}\text { R.A. } \\
\text { J2000.0 } \\
\text { (hh mm ss.s) }\end{array}$ & $\begin{array}{c}\text { Decl. } \\
\text { J2000.0 } \\
\text { (+/-dd mm ss.ss) }\end{array}$ & Redshift & Distance & $\begin{array}{c}\text { uGMRT } \\
\text { Phase cal } \\
\text { (IAU name) }\end{array}$ & $\begin{array}{c}\text { uGMRT } \\
\text { Phase cal } \\
\text { flux density } \\
\text { (Jy) }\end{array}$ & $\begin{array}{l}\text { VLA Project } \\
\text { ID }\end{array}$ & $\begin{array}{c}\text { VLA } \\
\text { telecope } \\
\text { configuration }\end{array}$ & $\begin{array}{c}\text { VLA } \\
\text { frequency } \\
(\mathrm{GHz})\end{array}$ \\
\hline PG $0003+199$ & 000619.5 & +201210.49 & 0.02578 & 103 & $2330+110$ & 1.28 & AL0418 & $\mathrm{C}$ & 8 \\
\hline PG $0007+106$ & 001031.0 & +105829.50 & 0.08934 & 387 & $2330+110$ & 1.28 & AF0350 & $\mathrm{C}$ & 5 \\
\hline PG 0049+171 & 005154.7 & +172558.50 & 0.06400 & 271 & $0119+321$ & 3.47 & AL0418 & $\mathrm{C}$ & 8 \\
\hline PG $0050+124$ & 005334.9 & +124136.20 & 0.05890 & 248 & $0059+001$ & 3.51 & AK0298 & $\mathrm{C}$ & 5 \\
\hline PG 0923+129 & 092603.2 & +124403.63 & 0.02915 & 127 & $0842+185$ & 1.07 & AK0298 & $\mathrm{C}$ & 5 \\
\hline PG 0934+013 & 093701.0 & +010543.48 & 0.05034 & 220 & 0943-083 & 3.84 & AF0327 & $\mathrm{C}$ & 8 \\
\hline PG 1011-040 & 101420.6 & -041840.30 & 0.05831 & 256 & 0943-083 & 3.84 & AS0455 & $\mathrm{AB}$ & 8 \\
\hline PG $1119+120$ & 112147.1 & +114418.26 & 0.05020 & 219 & $1120+143$ & 3.66 & AK0298 & $\mathrm{C}$ & 5 \\
\hline PG $1211+143$ & 121417.6 & +140313.10 & 0.08090 & 358 & $1254+116$ & 0.89 & AK0298 & $\mathrm{C}$ & 5 \\
\hline PG 1229+204 & 123203.6 & +200929.21 & 0.06301 & 276 & $1254+116$ & 0.89 & AK0298 & $\mathrm{C}$ & 5 \\
\hline PG 1244+026 & 124635.2 & +022208.79 & 0.04818 & 210 & $1254+116$ & 0.89 & AF0327 & $\mathrm{C}$ & 8 \\
\hline PG $1310-108$ & 131305.7 & -110742.40 & 0.03427 & 149 & $1248-199$ & 7.84 & AK0298 & $\mathrm{C}$ & 5 \\
\hline PG $1351+236$ & 135406.4 & +232549.09 & 0.05500 & 239 & $1330+251$ & 10.99 & AF0327 & $\mathrm{C}$ & 8 \\
\hline PG 1404+226 & 140621.8 & +222346.22 & 0.09800 & 437 & $1330+251$ & 10.99 & AK0298 & $\mathrm{C}$ & 5 \\
\hline PG 1426+015 & 142906.5 & +011706.48 & 0.08657 & 383 & $1445+099$ & 2.34 & AK0298 & $\mathrm{C}$ & 5 \\
\hline PG 1448+273 & 145108.7 & +270926.92 & 0.06500 & 283 & $3 C 286$ & 22.51 & AK0298 & $\mathrm{C}$ & 5 \\
\hline PG $1501+106$ & 150401.2 & +102616.15 & 0.03642 & 157 & $1445+099$ & 2.34 & AK0298 & $\mathrm{C}$ & 5 \\
\hline PG 2130+099 & 213227.8 & +100819.46 & 0.06298 & 266 & $2148+069$ & 2.81 & AL0418 & $\mathrm{C}$ & 8 \\
\hline PG 2209+184 & 221153.8 & +184149.86 & 0.07000 & 298 & $2148+069$ & 2.81 & AL0418 & $\mathrm{C}$ & 8 \\
\hline PG 2214+139 & 221712.2 & +141420.89 & 0.06576 & 279 & $2148+069$ & 2.81 & AG0173 & B & 1.5 \\
\hline PG 2304+042 & 230702.9 & +043257.22 & 0.04200 & 173 & $2330+110$ & 1.28 & AL0418 & $\mathrm{C}$ & 8 \\
\hline
\end{tabular}

sitivity falls significantly beyond this range. Therefore, we omitted the bad channels and non-working antennas in the log file produced from LISTSCAN so as to produce a FITS file of the frequency range 560-810 MHz. The FITS file was then converted to Measurement Set (MS) using the task IMPORTUVFITS in CASA. Subsequent flagging of data affected by radio frequency interference (RFI) at various stages were carried out using TFCROP and RFLAG modes of task FLAGDATA in CASA. Manual flagging of all calibrators and individual target sources using PLOTMS in CASA significantly improved the data.

The calibration steps involved were: setting flux density values for amplitude calibrators, initial phase calibration to correct for phase variations with time in the bandpass, delay calibration to correct for antenna-based delays, bandpass calibration to correct for bandpass shapes and gain calibration to correct for complex antenna gains. The flux density values for amplitude calibrators were set using the task SET JY in CASA and were determined for phase calibrators using the task FLUXSCALE in CASA. The output of SETJY was $32.18 \mathrm{Jy}$ for 3C 48 and 22.51 Jy for 3C 286 (using Perley-Butler 2017 scale). The output of FLUXSCALE is listed in Table 1. The calibrator solutions were then applied to the multisource target data set. The CASA task SPLIT was used to create visibility subsets for individual targets from the original data set while averaging the spectral channels such that the bandwidth smearing effects were negligible. Each target data set was finally imaged using the wide-band, wide-field imaging algorithm of TCLEAN task in CASA. Faceted imaging was combined with multiterm imaging to achieve this (specmode $=\mathrm{mfs}$; deconvolver $=$ mtmfs; gridder $=$ widefiled). Several iterations of phase-only self-calibration and minimum two iterations of amplitude and phase self-calibration until convergence were per- formed to improve the sensitivity of the images ${ }^{2}$. The final synthesized beam of the images is typically $\sim 3-5^{\prime \prime}$. The largest angular scale that can be measured through these observations is $\sim 25^{\prime}$.

\subsection{Data reduction and imaging for PG 2209+184}

The total intensity image for PG 2209+184 obtained using the standard procedures in CASA showed a lot of imaging artefacts. It neither improved with manual flagging nor with sub-band imaging. Therefore, we analysed this source using SPAM ${ }^{3}$. SPAM cannot be directly run on GWB (GMRT Wideband Backend) data with a bandwidth of $400 \mathrm{MHz}$. Therefore, first, the GWB data were divided into subbands of data with bandwidth of $32 \mathrm{MHz}$, which is equal to the historical GSB (GMRT Software Backend) bandwidth. This was done while converting the LTA file to FITS file by editing the log file produced from LISTSCAN. Then, SPAM was run on each subband data set independently, resulting in individual images from each data set. Several methods were tried to combine these images to yield one final image but none gave reasonable results, hence the sub-band UV data sets were combined. SPAM runs in 2 parts - (i) basic calibration; (ii) imaging. The first part ('pre_calibrate_targets') yields a file with extension CAL.RR.UVFITS, which is a calibrated stokes 'RR' visibility data. We had multiple CAL.RR.UVFITS files from the different sub-band data sets, which were first converted to MS files by running the CASA task IMPORTGMRT. Then, the multiple MS files were combined into a single MS file (taking two at a time) by running the CASA task CONCAT. Finally, the combined MS file

\footnotetext{
2 A Python script for reducing the total and polarized intensity uGMRT data is available at https://sites.google.com/view/silpasasikumar/

3 Source Peeling and Atmospheric Modeling; Intema (2014)
} 
was imaged using TCLEAN task in CASA. Also, before running SPAM, three of the python scripts had to be edited to match with the GWB frequency ranges and calibrator scan lengths. An intriguing observation was the presence of extended radio emission on few tens of kpc-scales in the final image, which was not visible above noise in the individual images obtained from each sub-band data set.

\subsection{Radio spectral index images}

The spectral index images and spectral index noise images were made using the task COMB in AIPS ${ }^{4}$. For this, we first obtained similar resolution ( 3-5") GHz-frequency raw visibility data from VLA Data Archive for 20 PG sources, excluding PG 0007+106 and PG 1341+258, which are discussed individually below (see Table 1 for VLA data details). The raw visibilities were first calibrated by running the task VLARUN in AIPS, followed by imaging using the task IMAGR in AIPS. Since, no flux calibrator was observed in the Projects - AK0298 and AG0173, we manually set it during the task VLARUN as the brightest phase calibrator from the respective projects. Also, their flux densities, taken from the VLA calibrator list ${ }^{5}$ at respective frequencies, were set for the parameter FLUX in the task VLARUN. The flux densities of the phase calibrators IAU 1252+119 (Project ID - AF0327), IAU 0922+005 (Project ID AF0327) and IAU 2121+053 (Project ID - AL0418) obtained from the task GETJY in VLARUN did not agree with the values given in the VLA calibrator list at respective frequencies. However, these calibrators are suggested to be variable in the literature (Stannard \& Bentley 1977; Fanti et al. 1981; Hughes et al. 1992; Torniainen et al. 2005).

For PG $0007+106$, similar resolution calibrated visibility data were obtained from VLA Image Archive and imaged using the task IMAGR in AIPS. Self-calibration failed for most of the faint sources (flux densities $\lesssim 2 \mathrm{mJy}$ ) and was therefore not carried out. The images of the sources at both frequencies (uGMRT and VLA) were then convolved with identical circular beams while setting the ROBUST parameter in IMAGR appropriately (ROBUST $=+5$ to -5 for the higher and lower resolution images, respectively). Also, the uGMRT and VLA images were made spatially coincident using the task OGEOM in AIPS. Finally, the spectral index images were created using the task COMB in AIPS. This task gives a 'two-point' spectral index for each pixel using the relation: $\alpha=\log \left(\mathrm{S}_{1} / \mathrm{S}_{2}\right) / \log \left(v_{1} / v_{2}\right)$ for flux densities $S_{1}$ and $S_{2}$ at frequencies $v_{1}$ and $v_{2}$. In the present work, $v_{1}=685 \mathrm{MHz}$ and $v_{2}$ is given in Table 1 for individual sources. Regions with flux densities below three times the rms noise were blanked while using COMB. The visibility data for PG $1341+258$ were available neither in the VLA Data Archive nor in the VLA Image Archive; therefore we could not produce its spectral index image. However, we obtained its spectral index value using the total flux density at $5 \mathrm{GHz}$ from Kellermann et al. (1994) and at 685 $\mathrm{MHz}$ from the current work.

\section{RESULTS}

We present the results of our multifrequency radio study in the following subsections. Section 4.1 presents the global properties of the PG sample. Section 4.2 discusses the radio morphological features of individual sources. The later sections aim to determine the origin

4 Astronomical Image Processing System; Wells (1985)

5 https://science.nrao.edu/facilities/vla/observing/callist

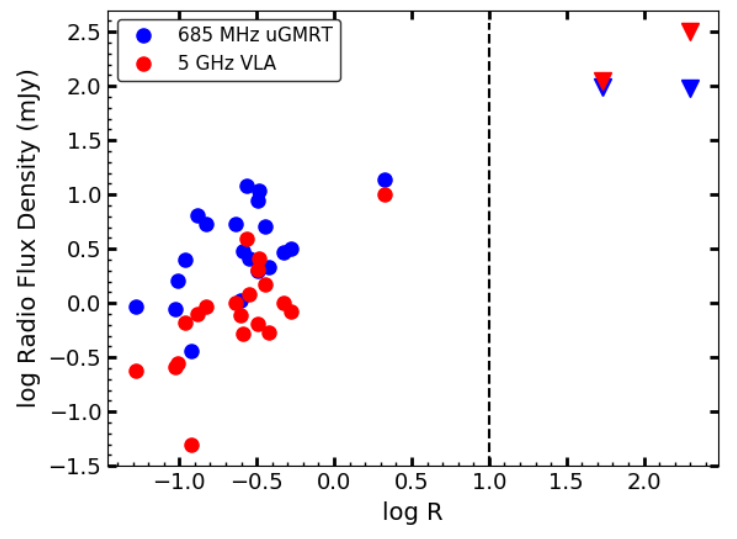

Figure 1. $685 \mathrm{MHz}$ uGMRT and $5 \mathrm{GHz}$ VLA flux density as a function of radio-loudness parameter for the PG sources. The values of $5 \mathrm{GHz}$ flux density and radio-loudness are taken from Kellermann et al. (1994). The black dashed vertical line divides the RL and RQ PG sources.

of radio emission based on spectral index analysis (Section 4.3), radio-IR correlation (Section 4.4) and correlations between radio and accretion properties (Section 4.5).

\subsection{Global properties of the PG sample}

The $685 \mathrm{MHz}$ uGMRT contour images and radio-optical overlay images created using grizy $^{6}$-color composite optical images taken from PanSTARRS (Flewelling et al. 2016) for individual sources (except PG 0007+106, which is provided in Figure 2) are presented in APPENDIX B. Small positional offsets (typically $<1^{\prime \prime}$ ) between the $685 \mathrm{MHz}$ uGMRT images and the optical images in seven sources were corrected using the HEASARC Fv software ${ }^{7}$. The $685 \mathrm{MHz}$ peak flux density of individual sources were estimated using the task TVMAXF in AIPS. It varies around a few mJy beam ${ }^{-1}$ to $\sim 100$ mJy beam ${ }^{-1}$ and the rms noise is around $\sim 20-40 \mu \mathrm{Jy}_{\text {beam }}{ }^{-1}$. The peak signal-to-noise ratio varies from $\sim 10$ to $\sim 1700$. The 685 MHz total flux density of all the sources, except PG 0007+106 and PG 1229+204, were estimated using the task JMFIT in AIPS. PG 0007+106 and PG 1229+204 have multiple components, therefore their total flux densities were estimated using the task TVSTAT in AIPS. The $685 \mathrm{MHz}$ total and peak flux densities for individual sources are presented in Table 2. The errors in the total flux densities were estimated from the calibration uncertainties (which is typically $\sim 10 \%$ for uGMRT) and rms noise of the images.

We present a discussion on the optical morphology of the PG quasar host galaxies in APPENDIX A, based on their classification in NED $^{8}$ (see Table 2), HST image analysis (Kim et al. 2008, 2017) and visual inspection of the PanSTARRS images. We find that the host galaxies of $50 \%$ of the sources show signatures of galaxy interactions, such as tidal tails, rings, or other isophotal distortions (see APPENDIX A and the radio-optical overlay images in APPENDIX B). However, this disturbed fraction could be a lower limit due to

${ }^{6}$ grizy is a set of five broadband filters used in PanSTARRS1 observations. The mean wavelengths of $\mathrm{g}, \mathrm{r}, \mathrm{i}, \mathrm{z}$ and $\mathrm{y}$ filters are $4866 \AA, 6215 \AA, 7545 \AA, 8679 \AA$ and $9633 \AA$ respectively (https://outerspace.stsci.edu/display/PANSTARRS/PS1+Filter+properties).

7 https://heasarc.gsfc.nasa.gov/docs/software/ftools/fv/

8 NASA/IPAC Extraglactic Database (https://ned.ipac.caltech.edu/) 
lack of detailed analyses/high-resolution imaging across the sample (for e.g., the HST image analysis is available for only five sources in our sample). We also find that the radio emission in $\sim 30 \%$ of these disturbed sources have AGN origin whereas the remaining $\sim 70 \%$ have both AGN and stellar origin (see Sections 4.3 and 4.4).

Figure 1 clearly depicts the RL-RQ dichotomy in the PG sample using the new $685 \mathrm{MHz}$ uGMRT data. The radio-loudness parameter values and $5 \mathrm{GHz}$ VLA flux densities are taken from Kellermann et al. (1994). We see that the $685 \mathrm{MHz}$ flux densities are higher than the $5 \mathrm{GHz}$ flux densities. This suggests that the uGMRT $685 \mathrm{MHz}$ observations have detected copious amounts of low frequency diffuse emission, missed out by the previous $5 \mathrm{GHz}$ VLA observations.

\subsection{Radio Morphology of Individual Sources}

In this Section, we briefly discuss the radio morphology of the PG sources. Nearly $80 \%$ sources are only a combination of a radio core unresolved at the uGMRT resolution of $\sim 3-5^{\prime \prime}$ surrounded by diffuse emission, together which we define as the 'uGMRT core' or equivalently the 'core' for our sources. The angular sizes of the cores are estimated using the Gaussian-fitting task JMFIT in AIPS. We present the redshift-dependent linear sizes of the radio cores of individual sources in Table 2. The error returned by the task JMFIT are taken as the error in the core size. The uGMRT cores of the PG sources are spatially resolved/barely resolved at $\sim 2-10 \mathrm{kpc}$ scales. PG 0050+124 and PG 1501+106 show double-sided diffuse emission on few kpc-scales. PG 2214+139 is the only unresolved source in our sample. Only four sources - PG 0007+106, PG 1229+204, PG 1244+026 and PG 1448+273 (discussed individually below) show extended radio emission on $\sim 10-30 \mathrm{kpc}$ scales. These sources extend beyond their host galaxies while the rest are largely confined to within their host galaxies.

The RL quasar PG 0007+106 (a.k.a. III Zw 2) shows the most extended (projected) radio structure at $685 \mathrm{MHz}$. A triple radio source is clearly detected. The core-hotspot distance is $\sim 30 \mathrm{kpc}$ on either side, with the south-western radio jet/lobe being on the approaching side. A misaligned system of low-frequency diffuse emission around the triple radio source is detected, which could be due to a previous episode of AGN activity or triggered by some merger event and misaligned due to a change in the jet direction. We present its lower resolution image in Figure 2, which was produced by $u v$-tapering the visibility data at $15 \mathrm{k} \lambda$, in order to fully capture the diffuse extended emission. The $685 \mathrm{MHz}$ peak flux density, rms noise and radio core size for PG $0007+106$ reported in Table 2 were estimated from the original resolution image (i.e. before $u v$-tapering). Also, the $685 \mathrm{MHz}$ total flux density reported in Table 2 and used in the calculation of luminosities, pertain to the hotspot-core-hotspot structure in the original resolution image and does not include the contribution of the misaligned lobe emission. Moreover, we find that the $685 \mathrm{MHz}$ total flux density inclusive of the misaligned lobe emission estimated from the $u v$-tapered image of PG $0007+106$ using the task TVSTAT in AIPS is $\sim 120 \mathrm{mJy}$.

Similar tapering of the visibility data in compact PG sources failed to detect any large-scale lobe-like diffuse emission. This suggests that our current uGMRT observations are not sensitive enough to detect $\sim 100 \mathrm{kpc}$-scale emission of the type detected in the $\mathrm{X}$ shaped radio galaxy NGC 326 with Low-Frequency Array (LOFAR) (Hardcastle et al. 2019). Alternately, there is no large-scale remnant 'relic' emission in the compact PG sources.

PG 1229+204 appears as a V-shaped triple radio source (hotspot-core-hotspot; see e.g., Kharb et al. 2012) comprising of a $\sim$ kpc-scale extended core in the NE-SW direction and a $\sim 0.1$ mJy component ( $\sim 3 \sigma$ feature) at a distance of $\sim 15 \mathrm{kpc}$ in the NW direction. This V-shaped morphology is possibly arising from the projection effects at small viewing angles. The radio morphology of PG 1244+026 resembles kpc-scale or 8-shaped radio structures in Seyfert galaxies (Gallimore et al. 2006). A core with extended emission to the west in PG 1448+273 resembles one-sided core-jet structure.

Figure 3 reveals no correlation between the radio core size and the mean core spectral index, denoted by $\alpha_{R}$ (see Section 4.3) for the RQ sources; the Spearman rank correlation co-efficient $r_{s}=0.256$ and probability $\mathrm{p}_{s}=0.277$. This could suggest that the uGMRT cores of the sources with steep spectra contain unresolved/barely resolved jets and lobes. The flat spectra in the cores could either suggest the presence of synchrotron self-absorbed jet bases or free-free emission from accretion disk winds on scales of 100s of parsecs or from the tori.

\subsection{Radio Spectral Indices: Implications for Source Structure}

Both stellar and AGN processes can contribute to radio emission in active galaxies. Each has a thermal and a non-thermal component. Although it is difficult to estimate the relative contributions of thermal and non-thermal emission, it is possible to infer the same from spectral index values. Thermal free-free radio emission from HII regions or accretion disc/torus has a spectral index of -0.1 , whereas non-thermal radio emission from starburst superwinds or AGN jets/AGN-driven winds has much steeper spectral index.

We present the spectral index images created using $685 \mathrm{MHz}$ uGMRT data and similar resolution ( 3-5") GHz-frequency VLA data in color superimposed with $685 \mathrm{MHz}$ total intensity contours for individual sources in APPENDIX B. $\alpha_{R}$ is the mean spectral index value of the uGMRT core of a source estimated from its spectral index image using the task IMSTAT or TVSTAT in AIPS and the error in $\alpha_{R}$ is the mean value from the same region of the spectral index noise image. The values of $\alpha_{R}$ for individual sources are provided in Table 3. The sources with $\alpha_{R}>0$ have inverted spectrum cores. We consider the sources with $\alpha_{R} \geq-0.5$ to have flat spectrum cores and the sources with $\alpha_{R}<-0.5$ to have steep spectrum cores.

The cores of the two RL sources, PG 0007+106 and PG $2209+184$ exhibit inverted radio spectrum. The inverted (and flat) spectrum cores in RL sources are expected to be the unresolved optically thick synchrotron self-absorbed bases of relativistic jets.

The jet and the south-western lobe of PG $0007+106$ has a steep spectrum, with hints of diffuse $\alpha_{R}<-1$ emission typically associated with 'relic' emission from a previous episode of AGN activity (e.g., Roettiger et al. 1994; Kharb et al. 2016). The southwestern lobe and the core have also been detected in the similar resolution VLA image, which is used in making the spectral index image. However, the north-eastern lobe and the misaligned lobe have not been detected in this VLA image. We suggest that their nondetection is indicative of steep spectrum lobe emission rather the lack of VLA sensitivity based on our following calculations. Using the rms noise in the VLA image and the intensity of the northeastern and the misaligned lobes in the uGMRT image, we find that the north-eastern and the misaligned lobes should have been detected with $\sim 15 \sigma$ and $\sim 3.8 \sigma$ significance respectively, if their spectrum was say -0.7 (which is a typical value for optically thin synchrotron emission). Assuming the VLA image was adequately sensitive, we took a $3 \sigma$ flux limit for the north-eastern and the misaligned lobes and obtained a limit on their spectral index values, 
Table 2. Summary of radio and optical properties of PG quasars

\begin{tabular}{|c|c|c|c|c|c|c|c|c|}
\hline Quasar & $\begin{array}{c}\text { Host galaxy* } \\
\text { morphology } \\
\text { (from NED)* }\end{array}$ & $\begin{array}{c}\text { Radio-loudness } \\
\text { R }\end{array}$ & $\begin{array}{l}\text { Classification } \\
\text { by radio }\end{array}$ & $\begin{array}{c}685 \mathrm{MHz} \text { peak } \\
\text { flux density } \\
\left(\mathrm{mJy} \mathrm{beam}^{-1}\right)\end{array}$ & $\begin{array}{c}685 \mathrm{MHz} \text { total } \\
\text { flux density } \\
\mathrm{S}_{685}(\mathrm{mJy})\end{array}$ & $\begin{array}{c}r m s \text { noise } \\
\left(\mu \mathrm{Jy} \mathrm{beam}^{-1}\right)\end{array}$ & $\begin{array}{l}\text { Radio core } \\
\text { resolved at } \\
685 \mathrm{MHz} ?\end{array}$ & $\begin{array}{l}\text { Radio core } \\
\text { size } \\
(\mathrm{kpc})\end{array}$ \\
\hline PG 0003+199 & $\mathrm{S} 0 / \mathrm{a}$ & 0.27 & RQ & 11 & $12 \pm 1$ & 31.68 & Yes & $2.578 \pm 0.006$ \\
\hline PG $0007+106$ & - & 200 & $\mathrm{RL}$ & 56 & $95 \pm 6$ & 33.47 & Yes & $10.107 \pm 0.004$ \\
\hline PG 0049+171 & - & 0.32 & $\mathrm{RQ}$ & 1.8 & $2.0 \pm 0.2$ & 64.24 & Yes & $5.4 \pm 0.2$ \\
\hline PG $0050+124$ & $\mathrm{Sa}$;Sbrst & 0.33 & RQ & 9.5 & $11.0 \pm 1.0$ & 34.54 & Yes & $4.71 \pm 0.01$ \\
\hline PG $0923+129$ & S0? & 2.1 & RQ & 12 & $14 \pm 1$ & 27.77 & Yes & $5.69 \pm 0.01$ \\
\hline PG 0934+013 & SBab & 0.38 & RQ & 1.2 & $2.1 \pm 0.1$ & 23.26 & Yes & $8.0 \pm 0.2$ \\
\hline PG 1011-040 & SB(r)b: pec & 0.097 & $\mathrm{RQ}$ & 1.2 & $1.6 \pm 0.1$ & 22.66 & Yes & $9.4 \pm 0.2$ \\
\hline PG $1119+120$ & SABa & 0.15 & RQ & 3.2 & $5.4 \pm 0.3$ & 29.11 & Yes & $7.67 \pm 0.06$ \\
\hline PG $1211+143$ & - & 0.13 & RQ & 4.7 & $6.5 \pm 0.5$ & 155.3 & Yes & $7.78 \pm 0.06$ \\
\hline PG 1229+204 & SB0 & 0.11 & $\mathrm{RQ}$ & 1.9 & $2.5 \pm 0.2$ & 28.71 & Yes & $6.03 \pm 0.08$ \\
\hline PG $1244+026$ & $\mathrm{E} / \mathrm{S} 0$ & 0.53 & RQ & 2.9 & $3.1 \pm 0.3$ & 26.48 & Maybe & $5.14 \pm 0.04$ \\
\hline PG $1310-108$ & - & 0.095 & RQ & 0.74 & $0.87 \pm 0.08$ & 30.64 & Yes & $3.2 \pm 0.1$ \\
\hline PG $1341+258$ & - & 0.12 & $\mathrm{RQ}$ & 0.30 & $0.36 \pm 0.04$ & 23.85 & Yes & $6.7 \pm 0.5$ \\
\hline PG $1351+236$ & - & 0.26 & $\mathrm{RQ}$ & 2.1 & $3.0 \pm 0.2$ & 23.41 & Yes & $4.56 \pm 0.05$ \\
\hline PG $1404+226$ & - & 0.47 & RQ & 2.8 & $2.9 \pm 0.3$ & 20.18 & Maybe & $6.83 \pm 0.04$ \\
\hline PG $1426+015$ & E/S0 & 0.28 & RQ & 2.0 & $2.6 \pm 0.2$ & 24.26 & Maybe & $7.61 \pm 0.09$ \\
\hline PG $1448+273$ & - & 0.23 & $\mathrm{RQ}$ & 5.3 & $5.3 \pm 0.5$ & 26.97 & Maybe & $6.23 \pm 0.03$ \\
\hline PG $1501+106$ & E & 0.36 & RQ & 3.7 & $5.1 \pm 0.4$ & 204.3 & Yes & $3.72 \pm 0.08$ \\
\hline PG 2130+099 & (R)Sa & 0.32 & RQ & 7.5 & $8.7 \pm 0.7$ & 24.44 & Yes & $4.93 \pm 0.01$ \\
\hline PG 2209+184 & - & 54 & RL & 93 & $97 \pm 9$ & 95.48 & Yes & $7.010 \pm 0.004$ \\
\hline PG 2214+139 & - & 0.052 & $\mathrm{RQ}$ & 0.94 & $0.93 \pm 0.10$ & 25.46 & No & 1.0 \\
\hline PG 2304+042 & - & 0.25 & RQ & 1.0 & $1.1 \pm 0.1$ & 32.09 & Maybe & $4.6 \pm 0.2$ \\
\hline
\end{tabular}

${ }^{*}$ E=elliptical; S0=lenticular; S=spiral; Sbrst=starburst; pec=peculiar; ?=uncertainty; a,b represent the tightness of the spiral arms; A,B represent bars; (r) represents inner ring; (R) represents outer ring. ${ }^{* *}$ see APPENDIX A for the references

Table 3. Summary of physical properties of PG quasars

\begin{tabular}{|c|c|c|c|c|c|c|c|c|c|}
\hline Quasar & $\alpha_{R}^{a}$ & $\begin{array}{c}\mathrm{L}_{685}^{b} \\
\left(10^{22} \mathrm{~W} \mathrm{~Hz}^{-1}\right)\end{array}$ & $\begin{array}{c}\mathrm{L}_{1400}{ }^{c} \\
\left(10^{22} \mathrm{~W} \mathrm{~Hz}^{-1}\right)\end{array}$ & $\begin{array}{c}\mathrm{L}_{5000}{ }^{d} \\
\left(10^{28} \mathrm{erg} \mathrm{s}^{-1} \mathrm{~Hz}^{-1}\right)\end{array}$ & $\begin{array}{l}\mathrm{L}_{\mathrm{IR}, \text { host }} e \\
\left(10^{11} \mathrm{~L}_{\odot}\right)\end{array}$ & $\mathrm{q}_{\mathrm{IR}}$ & $\begin{array}{c}\log \mathrm{L}_{\mathrm{X}}^{f} \\
\left(\operatorname{erg~s}^{-1}\right)\end{array}$ & $\begin{array}{c}\log \mathrm{M}_{\mathrm{BH}} g \\
\left(\mathrm{M}_{\odot}\right)\end{array}$ & $\begin{array}{c}\text { Edd. } \\
\text { ratio }\end{array}$ \\
\hline PG $0003+199$ & $-0.77 \pm 0.05$ & $1.5 \pm 0.1$ & $0.88 \pm 0.09$ & $3.4 \pm 0.5$ & 0.02 & $1.36 \pm 0.04$ & 43.80 & 7.52 & 0.21 \\
\hline PG 0007+106 & $+0.06 \pm 0.04$ & $155 \pm 9$ & $161 \pm 11$ & $1732 \pm 174$ & 0.48 & $0.48 \pm 0.03$ & 44.49 & 8.87 & 0.05 \\
\hline PG 0049+171 & $-0.46 \pm 0.10$ & $1.7 \pm 0.2$ & $1.2 \pm 0.1$ & $7 \pm 1$ & 0.03 & $1.39 \pm 0.05$ & 44.05 & 8.45 & 0.02 \\
\hline PG $0050+124$ & $-1.1 \pm 0.1$ & $8.1 \pm 0.7$ & $3.8 \pm 0.5$ & $10 \pm 3$ & 2.93 & $2.89 \pm 0.06$ & 44.18 & 7.57 & 2.26 \\
\hline PG $0923+129$ & $-0.72 \pm 0.06$ & $2.6 \pm 0.2$ & $1.6 \pm 0.2$ & $6.5 \pm 0.9$ & 0.22 & $2.15 \pm 0.04$ & 43.69 & 7.52 & 0.12 \\
\hline PG 0934+013 & $-0.5 \pm 0.1$ & $1.21 \pm 0.07$ & $0.85 \pm 0.09$ & $4 \pm 1$ & 0.25 & $2.48 \pm 0.05$ & 43.73 & 7.15 & 0.24 \\
\hline PG 1011-040 & $-0.47 \pm 0.08$ & $1.24 \pm 0.10$ & $0.89 \pm 0.08$ & $4.9 \pm 0.8$ & 0.28 & $2.50 \pm 0.04$ & 42.60 & 7.43 & 0.27 \\
\hline PG $1119+120$ & $-0.62 \pm 0.09$ & $3.0 \pm 0.2$ & $2.0 \pm 0.2$ & $9 \pm 2$ & 0.36 & $2.27 \pm 0.04$ & 43.48 & 7.58 & 0.32 \\
\hline PG $1211+143$ & $-0.59 \pm 0.07$ & $9.7 \pm 0.7$ & $6.4 \pm 0.6$ & $31 \pm 5$ & 0.00 & $\ldots$ & 44.54 & 8.10 & 0.46 \\
\hline PG 1229+204 & $-0.4 \pm 0.1$ & $2.2 \pm 0.2$ & $1.6 \pm 0.2$ & $10 \pm 3$ & 0.26 & $2.20 \pm 0.06$ & 44.00 & 8.26 & 0.08 \\
\hline PG $1244+026$ & $-0.7 \pm 0.1$ & $1.6 \pm 0.1$ & $1.0 \pm 0.1$ & $4.3 \pm 0.9$ & 0.16 & $2.20 \pm 0.05$ & 43.58 & 6.62 & 1.15 \\
\hline PG 1310-108 & $-0.5 \pm 0.2$ & $0.23 \pm 0.02$ & $0.16 \pm 0.03$ & $0.8 \pm 0.3$ & 0.04 & $2.41 \pm 0.07$ & 43.30 & 7.99 & 0.04 \\
\hline PG $1341+258$ & $-1.00 \pm 0.05$ & $0.64 \pm 0.07$ & $0.32 \pm 0.04$ & $0.9 \pm 0.1$ & 0.22 & $2.85 \pm 0.05$ & 43.83 & 8.15 & 0.06 \\
\hline PG $1351+236$ & $-1.0 \pm 0.2$ & $2.0 \pm 0.1$ & $1.0 \pm 0.1$ & $2.6 \pm 0.8$ & 0.50 & $2.71 \pm 0.06$ & 43.11 & 8.67 & 0.006 \\
\hline PG $1404+226$ & $-0.6 \pm 0.1$ & $6.4 \pm 0.6$ & $4.3 \pm 0.6$ & $21 \pm 6$ & 0.21 & $1.69 \pm 0.06$ & 43.77 & 7.01 & 0.95 \\
\hline PG $1426+015$ & $-0.49 \pm 0.10$ & $4.3 \pm 0.3$ & $3.1 \pm 0.3$ & $16 \pm 3$ & 0.51 & $2.23 \pm 0.04$ & 44.58 & 9.15 & 0.04 \\
\hline PG $1448+273$ & $-0.71 \pm 0.06$ & $5.0 \pm 0.5$ & $3.1 \pm 0.3$ & $12 \pm 2$ & 0.23 & $1.88 \pm 0.05$ & 43.86 & 7.09 & 0.95 \\
\hline PG $1501+106$ & $-0.6 \pm 0.1$ & $1.5 \pm 0.1$ & $1.0 \pm 0.1$ & $4.4 \pm 1.0$ & 0.20 & $2.32 \pm 0.05$ & 43.65 & 8.64 & 0.02 \\
\hline PG 2130+099 & $-0.65 \pm 0.06$ & $7.2 \pm 0.6$ & $4.6 \pm 0.4$ & $20 \pm 3$ & 0.50 & $2.04 \pm 0.04$ & 44.35 & 8.04 & 0.31 \\
\hline PG 2209+184 & $+0.05 \pm 0.03$ & $96 \pm 9$ & $100 \pm 10$ & $1064 \pm 119$ & 0.16 & $0.21 \pm 0.04$ & 43.94 & 8.89 & 0.006 \\
\hline PG 2214+139 & $-0.6 \pm 0.3$ & $0.84 \pm 0.09$ & $0.6 \pm 0.1$ & $3 \pm 2$ & 0.10 & $2.2 \pm 0.1$ & 42.63 & 8.68 & 0.03 \\
\hline PG 2304+042 & $-0.1 \pm 0.1$ & $0.37 \pm 0.04$ & $0.34 \pm 0.04$ & $2.8 \pm 0.6$ & 0.001 & $0.48 \pm 0.05$ & 43.89 & 8.68 & 0.003 \\
\hline
\end{tabular}

${ }^{a}$ Mean core spectral index. ${ }^{b} 685 \mathrm{MHz}$ uGMRT k-corrected rest-frame luminosity. ${ }^{c} 1400 \mathrm{MHz}$ k-corrected rest-frame luminosity derived using equations 2 and 3. ${ }^{d} 5000 \mathrm{MHz}$ k-corrected rest-frame luminosity derived using equations 2 and 3. ${ }^{e} 8-1000 \mu \mathrm{m}$ host galaxy IR luminosity from Lyu et al. (2017). ${ }^{f}$ $0.2-20 \mathrm{keV}$ X-ray luminosity from Laor \& Behar (2008). ${ }^{g} \mathrm{BH}$ mass from Shangguan et al. (2018). ${ }^{h}$ Eddington ratio (L $\mathrm{L}_{\mathrm{bol}} / \mathrm{L}_{\mathrm{Edd}}$ ) estimated using $\mathrm{M}_{\mathrm{BH}}$ and $\mathrm{L}_{\text {bol }}$ from Lyu et al. (2017). 

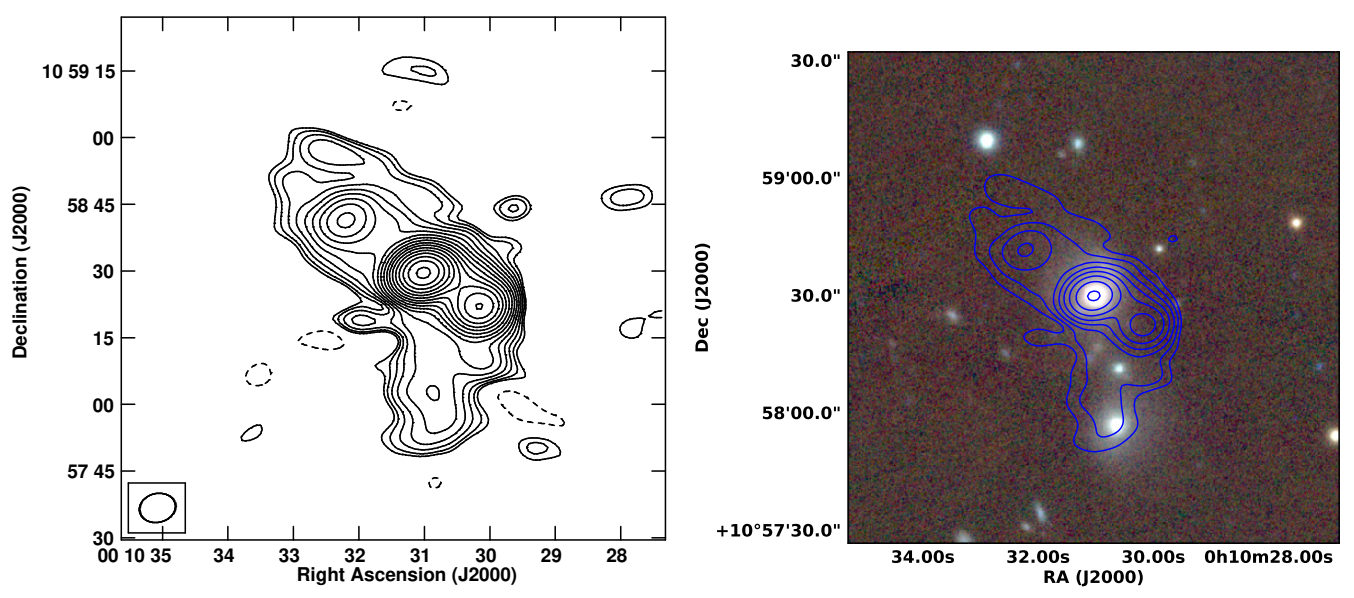

Figure 2. Left: $685 \mathrm{MHz}$ uGMRT total intensity contour image of RL PG quasar PG $0007+106$. The peak contour flux is $59 \mathrm{mJy}^{\text {beam }}{ }^{-1}$ and the contour levels are $0.21 \times(-1,1,1.4,2,2.8,4,5.6,8,11.20,16,23,32,45,64,90,128,180,256,362,512)$ mJy beam $^{-1}$. Right: $685 \mathrm{MHz}$ total intensity contours in blue superimposed on PanSTARRS grizy-color composite optical image of PG $0007+106$. The peak contour flux is $59 \mathrm{mJy}^{\text {beam }}{ }^{-1}$ and the contour levels are $0.21 \times(2,4,8,16,32,64,128,256,512) \mathrm{mJy}_{\text {beam }}{ }^{-1}$.

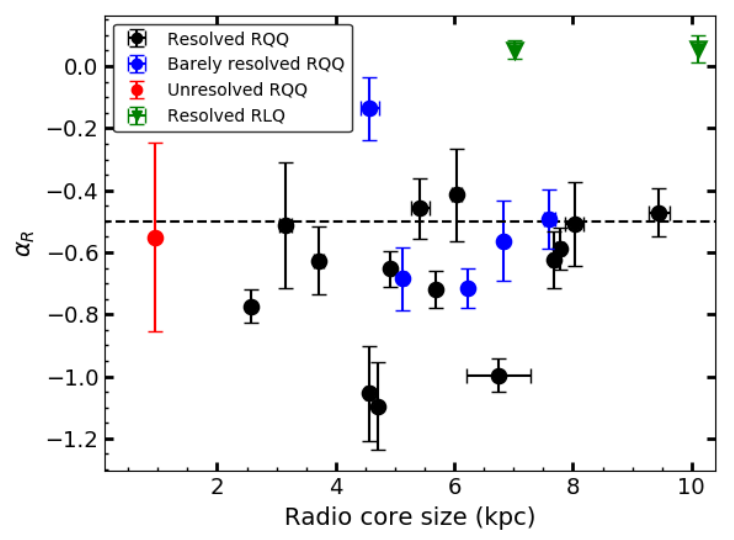

Figure 3. The mean core spectral index as a function of the uGMRT core size for the PG sources. The black dashed horizontal line marks $\alpha_{R}=-0.5$. We consider the sources with $\alpha_{R} \geq-0.5$ to have flat spectrum cores and the sources with $\alpha_{R}<-0.5$ to have steep spectrum cores.

which turn out to be $\sim-1.5$ and -0.8 respectively. The former is suggestive of 'relic' emission in the north-eastern lobe. Brunthaler et al. (2005) also found that both south-western and north-eastern lobes have steep spectral indices of -0.57 to -1.15 . Also, it is worth noting that the lobe emission is not detected in the $\sim 0.5^{\prime \prime} 5 \mathrm{GHz}$ VLA image of Kellermann et al. (1994).

In PG 2209+184, there is no evidence of jets or lobes in the uGMRT image or signs of spectral steepening in the spectral index image. It is possibly confined in dense environments, causing a decrease in expansion losses and increase in radiative losses, therefore being RL and compact with self-absorbed inverted spectrum (e.g., in Cygnus A, Barthel \& Arnaud 1996). PG 2209+184 appears as an unresolved core with an elongated component in the $5 \mathrm{GHz}$ VLA image (resolution $\sim 0.5^{\prime \prime}$ ) of Kellermann et al. (1994).

The remaining 20 sources are RQ and their cores reveal a range of spectral indices from flat to steep $\left(-0.1 \gtrsim \alpha_{R} \gtrsim-1.1\right)$, as was also found in Chiaraluce et al. (2020) for a sample of hard-X-rayselected AGN, although at higher frequencies. PG 0049+171, PG
1011-040, PG 1229+204, PG 1426+015 and PG 2304+042 have flat spectrum cores. PG $0934+013$ and PG 1310-108 are at the cusp of flat-steep division. There is no evidence of jets or lobes in the uGMRT total intensity images of these sources except PG 1229+204 (see Section 4.2). The transition of uGMRT core spectral index from flat to steep in the SE-NW direction in the spectral index image of PG $1229+204$, aligns with the double radio structure (possibly a corejet/lobe structure $)$ in the higher resolution $\left(\sim 0.5^{\prime \prime}\right)$ VLA image (Kellermann et al. 1994). PG 2304+042 appears as an unresolved core with an elongated component in the higher resolution $\left(\sim 0.5^{\prime \prime}\right)$ VLA image (Kellermann et al. 1994). PG 0049+171, PG 0934+013 and PG 1011-040 are unresolved in their higher resolution $\left(\sim 0.5^{\prime \prime}\right)$ VLA images (Kellermann et al. 1994). This means that the diffuse emission detected by uGMRT in these sources is being resolved out by the VLA observations. These RQ flat cores can be interpreted as being the unresolved self-absorbed bases of low-powered radio jets (on milliarcsec or sub-arcsec scales) confined within the uGMRT cores or 'frustrated' radio jets inside dense environments (O'Dea et al. 1991). Within errors, the flat spectrum core in PG 2304+042 may also arise due to thermal free-free emission from the accretion disc winds or from the torus or HII regions.

The remaining $13 \mathrm{RQ}$ sources have steep spectrum cores. These are PG 0003+199, PG 0050+124, PG 0923+129, PG 1119+120, PG 1211+143, PG 1244+026, PG 1341+258, PG 1351+236, PG 1404+226, PG 1448+273, PG 1501+106, PG 2130+099 and PG $2214+139$. The steep spectrum cores are either due to unresolved/barely resolved jet/lobe emission or optically thin synchrotron emission from AGN-/starburst-driven winds (Hwang et al. 2018). Some of these sources that reveal signatures of unresolved/barely resolved jets and lobes in their spectral index images are discussed individually below. We could rule out the possibility of these sources being steep Compact Symmetric Objects (CSO; Wilkinson et al. 1994; Orienti 2016) as they are neither as compact $(<1 \mathrm{kpc})$ nor as powerful $\left(\mathrm{L}_{1400} \geq 10^{25} \mathrm{WHz}^{-1}\right)$ as CSOs.

The uGMRT core of PG 2130+099 is resolved into jet/lobe structure in the higher resolution $\left(\sim 0.5^{\prime \prime}\right)$ VLA image (Kellermann et al. 1994), suggesting a jet origin for its steep spectrum, instead of AGN-/starburst-driven winds. PG 0003+199, PG 0050+124, PG $1404+226$ and PG 1448+273 are unresolved in their higher resolution $\left(\sim 0.5^{\prime \prime}\right)$ VLA images (Kellermann et al. 1994), which means 

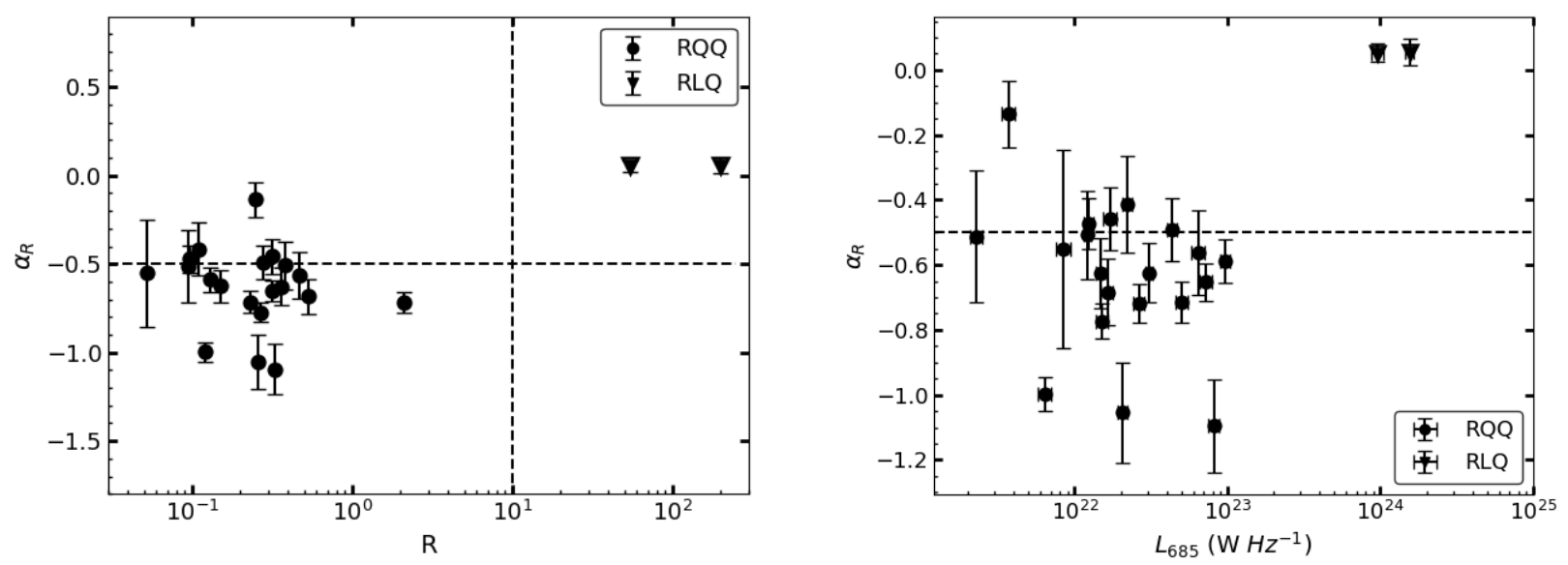

Figure 4. The mean core spectral index as a function of radio-loudness from Kellermann et al. (1994) (left) and 685 MHz k-corrected rest-frame luminosity for the PG sources (right). The black dashed horizontal line marks $\alpha_{R}=-0.5$. We consider the sources with $\alpha_{R} \geq-0.5$ to have flat spectrum cores and the sources with $\alpha_{R}<-0.5$ to have steep spectrum cores. The black dashed vertical line in the left panel divides the RL and RQ PG sources.

that the VLA observations resolve out the diffuse emission detected by uGMRT in these sources. Moreover, the absence of jet/lobe structures at this VLA resolution in these sources could suggest that their steep spectrum is either due to jet/lobe emission on even smaller spatial scales (milliarcsec or sub-arcsec scales) or due to AGN/starburst-driven winds. The total intensity images of PG 1244+026 and PG 1501+106 show kpc-scale extended emission (discussed in Section 4.2), which may suggest the presence of jet/lobe emission. However, this is not evident from their spectral index images, such as a gradient in their spectral index values away from the centre.

The spectral index image of PG 0050+124 suggests a barely resolved jet/lobe structure in the N-S direction. This coupled with $\alpha_{R} \sim-1$, may imply the presence of a pair of very steep lobes (may be 'relic') inside the uGMRT core. The spectral index image of PG $0923+129$ shows steep spectrum in the periphery of its core. This quasar is hosted by a ring galaxy. One of the suggested scenarios for the formation of ring galaxies is the merging of a very massive galaxy with a less massive one. The neighbouring galaxy in its radio-optical overlay image (see Figure B2 in APPENDIX B), seems to be its merger companion. This could suggest that the peripheral steep spectral emission arises from synchrotron radiation due to relativistic merger shocks (Ballard \& Heavens 1992). A gradient in the spectral index from flat to steep towards the east in the spectral index image of PG $1119+120$ could suggest a barely resolved jet/lobe structure in this direction. PG $1341+258$ and PG $1351+236$ have very steep spectrum of $\alpha_{R} \sim-1$, which could suggest the presence of unresolved lobe emission. An increasingly steeper spectral index towards the west coupled with a westward extension in the total intensity image of PG $1448+273$ could suggest a barely resolved one-sided jet/lobe structure.

Since one of the goals of the present study was to constrain the AGN duty cycle, we searched for signatures of diffuse 'relic' emission in the PG sources. However, we did not detect as much 'relic' emission as expected at low radio frequencies of the uGMRT. There are clear indications in one source - PG 0007+106, which is also supported by our calculations of the VLA sensitivity and missing flux. There are indications of the same in a few others based on their spectral index images.

Figure 4 (left-hand panel) reveals no correlation between $\alpha_{R}$ and the radio-loudness parameter $(\mathrm{R})$ for the RQ sources (Spearman rank correlation coefficient $r_{s}=-0.283$ and probability $\left.\mathrm{p}_{s}=0.227\right)$. Figure 4 (right-hand panel) reveals no correlation between $\alpha_{R}$ and the $685 \mathrm{MHz}$ k-corrected rest-frame luminosity $\left(\mathrm{L}_{685}\right)$ for the RQ sources $\left(r_{s}=-0.296, \mathrm{p}_{s}=0.205\right)$. However, we see two distinct regions being occupied by RL and RQ sources in these plots. We also find that while the RQ sources cover a range of spectral indices from flat to steep $\left(-0.1 \gtrsim \alpha_{R} \gtrsim-1.1\right)$, the (two) RL sources do not possess steep spectrum cores. Albeit small number statistics, this could suggest that the radio emission mechanisms in RQ AGN is intrinsically different to those in RL AGN, such as star formation (SF)/jets/winds in RQ AGN versus mainly jets in RL AGN.

We note that since the VLA and uGMRT observations were carried out at different epochs, variations in the flux density of the compact cores can affect the spectral index measurements. Barvainis et al. (2005) studied radio variability of a subset of PG quasars at $\sim 8 \mathrm{GHz}$. These included six sources from our sample, namely, PG 0003+199, PG 0007+106, PG 0049+171, PG 2130+099, PG $2209+184$ and PG 2304+042. We used $8 \mathrm{GHz}$ VLA data and $685 \mathrm{MHz}$ uGMRT data for making the spectral index images of PG 0003+199, PG 0049+171, PG 2130+099, PG 2209+184 and PG 2304+042. However, for PG 0007+106, we used $5 \mathrm{GHz}$ VLA data and $685 \mathrm{MHz}$ uGMRT data (see Table 1). Barvainis et al. (2005) found PG 0049+171, PG 2209+184 and PG 2304+042 to be variable. They estimated the maximum percentage change in the flux densities to be roughly $20 \%$ for PG $0049+171,20 \%$ for PG $2209+184$ and $100 \%$ for PG $2304+042$ over a time-scale of 0.5 , 0.1 and $0.5 \mathrm{yr}$, respectively. Using this information, we find that the spectral index value varies between -0.37 and -0.62 for PG $0049+171$ and between 0.1 and -0.8 for PG $2304+042$. For PG $2209+184$, the spectral index variation is of the order of 0.001 , much smaller than the estimated error in $\alpha_{R}$.

\subsection{The radio-IR correlation with uGMRT Data}

The correlation between radio and infrared (IR) emission is one of the tightest and well-studied correlations in astrophysics (Helou et al. 1985; Condon 1992; Yun et al. 2001; Bell 2003). It helps to identify whether the observed radio emission is dominated by AGN activity or SF in the host galaxy. A useful parameter that quantifies 


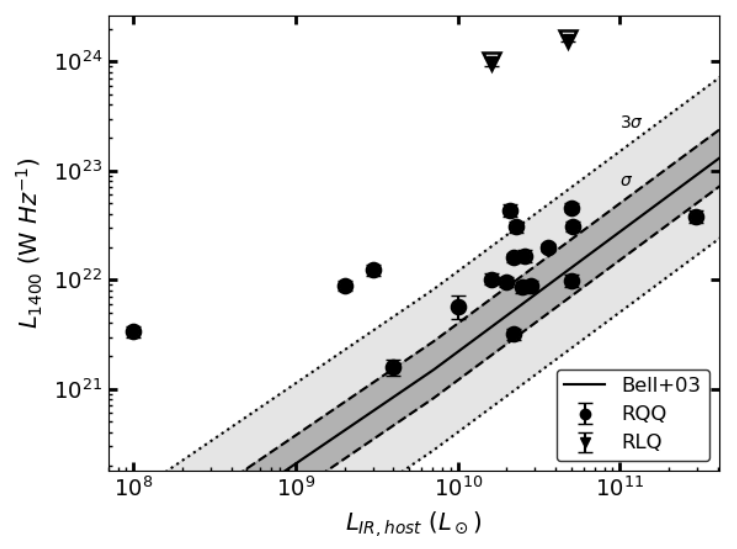

Figure 5. The radio-IR correlation for the PG sources. $\mathrm{L}_{1400}$ are the kcorrected rest-frame luminosities at $1400 \mathrm{MHz}$ derived from $685 \mathrm{MHz}$ uGMRT flux densities and $\alpha_{R}$ using equations 2 and 3 by substituting $v=1400 \mathrm{MHz}$. $\mathrm{L}_{\mathrm{IR} \text {, host }}$ are the $8-1000 \mu \mathrm{m}$ host galaxy IR luminosities taken from Lyu et al. (2017). The black solid line is the radio-IR correlation for star-forming galaxies from Bell (2003). The black dashed lines mark the $1 \sigma$ regions and the black dotted lines mark the $3 \sigma$ regions.

the radio-IR correlation is the qIR parameter (Bell 2003), which is defined as:

$\mathrm{q}_{\mathrm{IR}}=\log \left(\mathrm{L}_{\mathrm{IR}} / 3.75 \times 10^{12} \mathrm{~W}\right)-\log \left(\mathrm{L}_{1400} / \mathrm{W} \mathrm{Hz}^{-1}\right)$

The contribution of AGN to radio-IR correlation has been studied to some extent in the recent past. A low qIR value and a significant positional offset in the radio-IR correlation plane suggests the dominance of AGN in a galaxy. Condon et al. (2002) and Bell (2003) classify sources with qIR $_{1 \mathrm{~N}}<1.8$ as being "radio-excess" due to dominant contribution of AGN in their radio emission. The radio-IR correlation has been studied as a function of galaxy types in the literature. The average $\mathrm{q}_{\mathrm{IR}}$ values for star-forming galaxies, Seyfert galaxies and quasars are respectively 2.27, 2.14 and 2.04 (Morić et al. 2010). The qIR values for individual sources in our sample are listed in Table 3 . The average $\mathrm{q}_{\mathrm{IR}}$ value for the PG sample is $1.95 \pm 0.01$.

The flux density $\left(\mathrm{S}_{1400}\right)$ and the k-corrected rest-frame luminosity at $1.4 \mathrm{GHz}\left(\mathrm{L}_{1400}\right)$ for our sample are derived using the relations:

$\mathrm{S}_{v}=\mathrm{S}_{685}(v / 685)^{\alpha_{\mathrm{R}}}$

$\mathrm{L}_{v}=4 \pi \mathrm{D}_{\mathrm{L}}^{2} \mathrm{~S}_{v}(1+\mathrm{z})^{\left(-\alpha_{\mathrm{R}}-1\right)}$

where $v=1400 \mathrm{MHz}, \mathrm{S}_{685}$ are the observed uGMRT $685 \mathrm{MHz}$ total flux densities and $\alpha_{R}$ are the spectral index values taken from the images of individual sources. We use the 8-1000 $\mu \mathrm{m}$ IR luminosities ( $\mathrm{L}_{\mathrm{IR} \text {, host }}$ ) provided by Lyu et al. (2017), which include contributions from the host galaxy but not the AGN. Lyu et al. (2017) used SED fitting from optical to far-IR to isolate IR luminosity associated with SF in the host galaxy from that associated with AGN. Separating the IR contributions from the host galaxy and AGN is important because if both IR and radio emission are dominated by AGN, then this could produce another correlation that can artificially cause AGN to follow the radio-IR correlation characteristic of star-forming galaxies (Morić et al. 2010; Zakamska et al. 2016).

Figure 5 presents $\mathrm{L}_{1400}$ as a function of $\mathrm{L}_{\mathrm{IR} \text {,host }}$ for the PG sources and compares them with the radio-IR correlation of starforming galaxies from fig. 3 of Bell $(2003)^{9}$ :

$\log \mathrm{L}_{1400}\left(\mathrm{~W} \mathrm{~Hz}^{-1}\right)=1.10 \log \mathrm{L}_{\mathrm{IR}, \text { host }}\left(\mathrm{L}_{\odot}\right)+10.34$

The scatter in the radio-IR correlation of the star-forming galaxies in Bell (2003) is 0.26 dex, which was used to determine the $1 \sigma$ and $3 \sigma$ limits in Figure 5.

We see that both RL sources (PG 0007+106 \& PG 2209+184) and four RQ sources (PG 0003+199, PG 0049+171, PG 1404+226, PG 2304+042) clearly lie above the $3 \sigma$ limit of the radio-IR correlation. These sources also have $\mathrm{q}_{\mathrm{IR}}<1.8$. PG 1448+273 lies on the $3 \sigma$ limit and its $\mathrm{qIR}_{\mathrm{I}}$ is close to the AGN-SF discriminator value. PG $1211+143$ is not shown in Figures 5-7. For this source the optical to far-IR SED fitting in Lyu et al. (2017) indicated negligible contribution from host galaxy to the total IR luminosity compared to the AGN. This implies a negligible value of IR-based SFR following the Kennicutt (1998) SF law. The radio-based SFR can be taken as an upper limit to the SFR in this galaxy. Thus, consistent with Lyu et al. (2017), we conclude that AGN is the primary contributor to the radio emission in PG 1211+143.

Based on their position on the radio-IR correlation, we suggest that the radio emission in RL quasars and nearly one-third of the RQ quasars is AGN dominated. However, being on the radio-IR correlation does not rule out an AGN contribution in the remaining two-third of the RQ quasars (e.g., in Wong et al. 2016). In these sources, we suggest the possibility of a co-existence of AGN emission along with the stellar-related emission. There are signatures of unresolved/barely resolved jet/lobe structures on few arcsec-scales in the total intensity and the spectral index images of a few of these sources, such as, PG 0050+124, PG 1119+120, PG 1229+204, PG 1244+026, PG 1341+258, PG 1351+236, PG 1501+106 and PG 2130+099 (see Sections 4.2 and 4.3). Besides, the possibility of AGN outflows on milli-arcsec or sub-arcsec scales cannot be ruled out in the remaining sources. Moreover, we find that in $\sim 70 \%$ of our sample, the $1.4 \mathrm{GHz}$ luminosities estimated from the uGMRT 685 $\mathrm{MHz}$ data are significantly higher than those predicted from their radio-based SFRs following the Bell (2003) SF law, suggesting that the excess emission is associated with AGN. The radio-based SFRs have been assumed to be the same as the IR-based SFRs derived from $\mathrm{L}_{\mathrm{IR} \text {, host }}$ following the Kennicutt (1998) SF law.

Figure 6 provides $\mathrm{q}_{\mathrm{IR}}$ as a function of $\mathrm{L}_{1400}$ (top left panel), $\mathrm{L}_{\mathrm{IR} \text {,host }}$ (top right panel) and redshift (bottom panel) for the PG sources. Figure 6 (top left panel) and (bottom panel) reveals no correlation for the RQ PG sources based on the Spearman rank correlation test with $\left(r_{s} ; \mathrm{p}_{s}=-0.212 ; 0.383\right)$ and $\left(r_{s} ; \mathrm{p}_{s}=0.058\right.$ $; 0.814)$, respectively. However, in Figure 6 top right panel, we note an increase in the $\mathrm{q}_{I R}$ values with increasing $\mathrm{L}_{\mathrm{IR} \text {, host }}$ for the RQ PG sources, which is also implied as a positive correlation by the Spearman rank correlation test $\left(r_{s}=0.544\right.$ and $\left.\mathrm{p}_{s}=0.016\right)$. Figure 7 shows a $3 \sigma$ anticorrelation between $\mathrm{q}_{\mathrm{IR}}$ and $\alpha_{R}$ for the PG sources. This figure represents the distribution of flat and steep spectrum core PG sources across the AGN-star-formation divide ( $\left.\mathrm{qIR}_{\mathrm{IR}}=1.8\right)$. The best-fitting linear regression and the $1 \sigma$ and $3 \sigma$ limits are estimated using the LTS_LINEFIT program described in Cappellari et al. (2013). This program computes Spearman rank and Pearson

9 The slope and intercept were obtained from the figure using: Mark Mitchell, Baurzhan Muftakhidinov and Tobias Winchen et al, "Engauge Digitizer Software." Webpage: http://markummitchell.github.io/engaugedigitizer. 

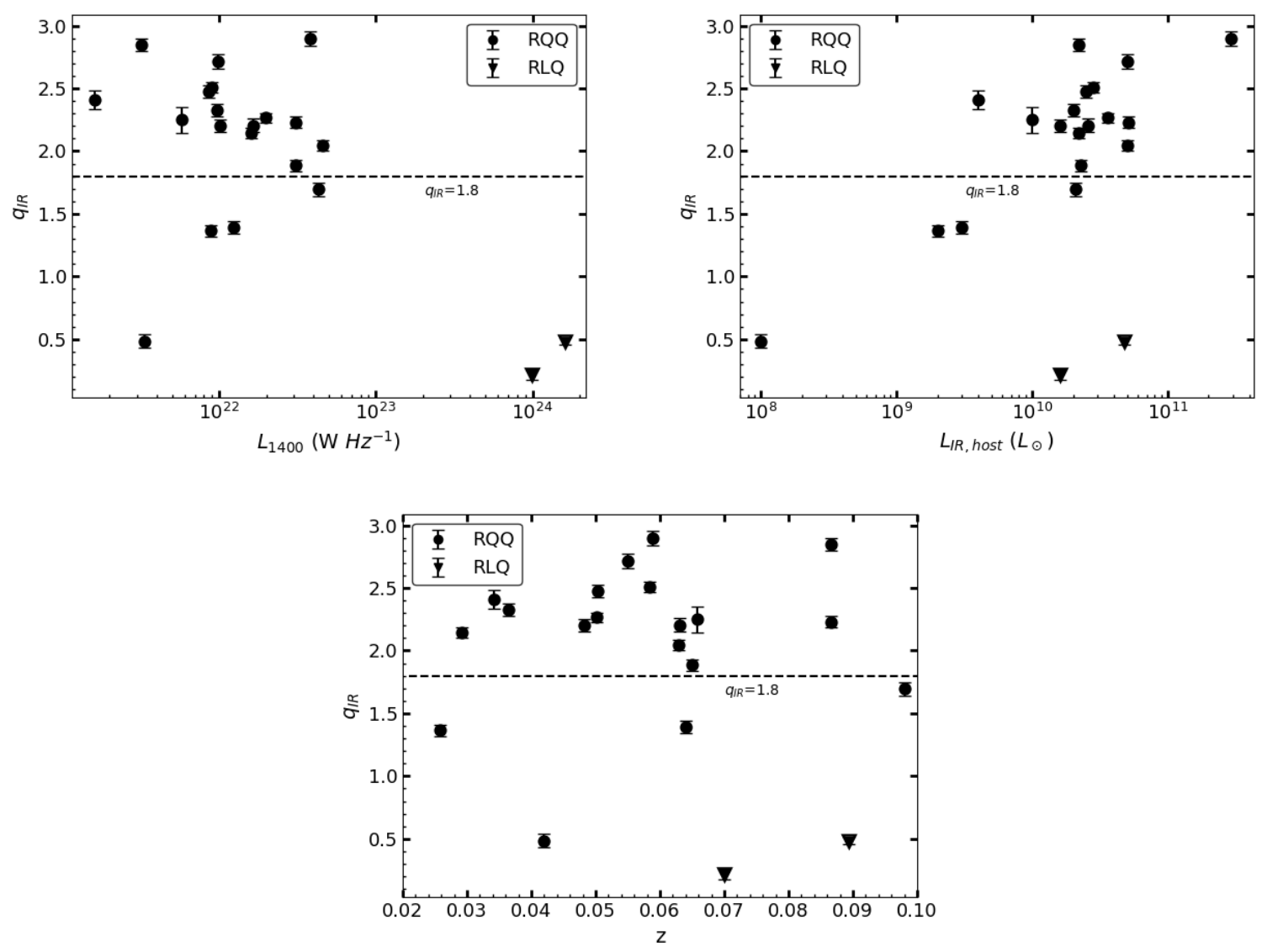

Figure 6. The $\mathrm{qII}_{\mathrm{IR}}$ as a function of $1400 \mathrm{MHz}$ k-corrected rest-frame luminosity derived from $685 \mathrm{MHz}$ uGMRT flux density and $\alpha_{R}$ using equations 2 and 3 by substituting $\nu=1400 \mathrm{MHz}$ (top left); 8-1000 $\mu$ m host galaxy IR luminosity taken from Lyu et al. (2017) (top right); and redshift for the PG sources (bottom). The black dashed line marks qIR $=1.8$, which is the discriminator value between AGN versus SF contributions to the observed radio emission (Condon et al. 2002; Bell 2003).

correlation co-efficients and does an iterative fit of the data assuming that the errors in both $\mathrm{x}$ - and $\mathrm{y}$ - co-ordinates are uncorrelated. It also returns the best fit slope and intercept values along with errors and plots the $1 \sigma$ and $3 \sigma$ limits based on the intrinsic scatter of the data. The Spearman rank and Pearson correlation tests imply an anticorrelation between $\mathrm{q}_{\mathrm{IR}}$ and $\alpha_{R}$ for our full PG sample with $r_{s}=$ -0.48 and $\mathrm{p}_{s}=0.027$ and Pearson correlation coefficient $r_{p}=-0.81$ and probability $\mathrm{p}_{p}=8.9 \times 10^{-6}$. However, for the RQ PG sample, only the Pearson test implies an anti-correlation $\left(r_{p}=-0.64\right.$ and $\mathrm{p}_{p}$ $=0.0033)$ whereas the Spearman test fails to show any correlation $\left(r_{s}=-0.3\right.$ and $\left.\mathrm{p}_{s}=0.21\right)$. Figure 7 is discussed below in detail:

(i) As discussed above, the radio emission in RL quasars and nearly one-third of the RQ quasars is dominated by AGN activity. Among these, the RL cores and two RQ cores (PG 0049+171 and PG 2304+042) have flat spectrum, possibly arising from synchrotron self-absorption or free-free emission from accretion disc/torus. The radio emission in the two RQ cores with steep spectrum (PG 0003+199 and PG 1404+226) may arise from AGN jets/AGNdriven winds. PG $1448+273$ which is at the cusp of AGN-SF divide, also has a steep spectrum core, possibly associated with AGN jets/AGN-driven winds. Morever, its total intensity image coupled with its spectral index image suggests AGN jet origin, instead of AGN winds for its steep spectrum (see Sections 4.2 and 4.3). Note that PG 1211+143, which is not indicated in the plot, also has a steep spectrum core, possibly arising from AGN jets/AGN-driven winds.

(ii) As mentioned earlier, the stellar-related emission is significant in two-third of the RQ quasars, based on their position on the radio-IR correlation and $\mathrm{q}_{\mathrm{IR}}$ values. Among these, three sources (PG 1011-040, PG 1229+204 and PG 1426+015) have flat spectrum cores possibly arising from thermal free-free emission around HII regions or mixing of the thermal emission with stellar-origin synchrotron emission, nine sources (PG 0050+124, PG 0923+129, PG 1119+120, PG 1244+026, PG 1341+258, PG 1351+236, PG 1501+106, PG 2130+099 and PG 2214+139) have steep spectrum cores most likely due to synchrotron-emitting shocks associated with starburst-superwinds and two sources (PG 0934+013 and PG 1310-108) are at the cusp of flat-steep division. However, the co-existence of AGN emission along with such stellar-related processes cannot be ruled out in these sources.

Using a sample of $\sim 300$ Type 1 and 2 quasars that included the complete PG sample, Zakamska et al. (2016) concluded that the radio emission in the quasars is dominated by AGN activity. They used 1.4 GHz flux densities from Faint Images of Radio Sky at Twenty cm survey (FIRST) and NRAO VLA Sky Survey (NVSS) and assumed a spectral index value of -0.7 to estimate the luminosities. 20 out of 22 sources from our sample are covered 


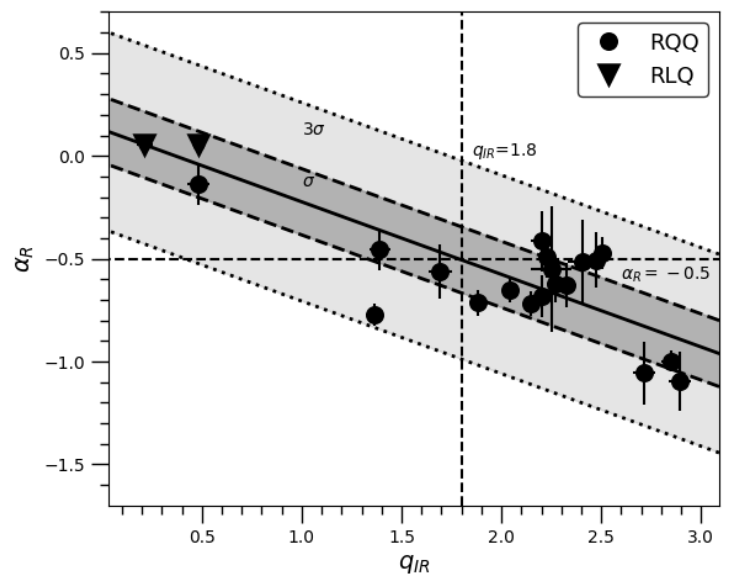

Figure 7. The mean core spectral index as a function of $\mathrm{q}_{\mathrm{IR}}$ for the PG sources. The black dashed vertical line marks $\mathrm{q}_{\mathrm{IR}}=1.8$, which is the discriminator value between AGN versus SF contributions to the observed radio emission (Condon et al. 2002; Bell 2003). The black dashed horizontal line marks $\alpha_{R}=-0.5$. We consider the sources with $\alpha_{R} \geq-0.5$ to have flat spectrum cores and the sources with $\alpha_{R}<-0.5$ to have steep spectrum cores. The black solid line is the best-fitting line for the data. The black dashed slant lines mark the $1 \sigma$ regions and the black dotted slant lines mark the $3 \sigma$ regions.

by FIRST and NVSS surveys. ${ }^{10}$ Note that the resolution of FIRST and NVSS is $5^{\prime \prime}$ and $45^{\prime \prime}$ respectively whereas the resolution of the uGMRT $685 \mathrm{MHz}$ images is $\sim 3-5^{\prime \prime}$. A comparison between the total $1.4 \mathrm{GHz}$ flux densities estimated from the uGMRT $685 \mathrm{MHz}$ data and those obtained from FIRST/NVSS reveals that $\sim 5-80 \%$ of the total flux density has been resolved out across 12 sources by the uGMRT observations. For half of the remaining eight sources, the total flux densities estimated from the uGMRT data are about two times higher than their respective FIRST/NVSS values, whereas for the rest, they are nearly the same. This coupled with Zakamska et al. (2016) work and the radio-IR correlation with uGMRT $685 \mathrm{MHz}$ data could suggest that the emission resolved out by the uGMRT observations is associated with AGN and the excess emission detected by the uGMRT observations is associated with star formation. This is in agreement with our previous suggestion that the low frequency $685 \mathrm{MHz}$ radio emission has significant contributions from both stellar-related processes and the AGN.

\subsection{Relation between radio properties and accretion properties}

\subsubsection{BH masses and Accretion rates}

The correlations between radio properties and $\mathrm{BH}$ properties in the PG sources have been discussed in the recent past. However, these studies were carried out using high-frequency $(\sim \mathrm{GHz})$ radio data. Using a combined sample of quasars from the FIRST Bright Quasar

10 Out of these 20, 11 sources are detected in FIRST and 3 sources are detected in NVSS. The remaining 6 sources are neither detected in NVSS nor FIRST. Therefore, for these sources, we compared the $1.4 \mathrm{GHz}$ peak flux densities estimated from the uGMRT $685 \mathrm{MHz}$ data with their $5 \sigma$ values, where $\sigma$ is the FIRST rms noise level at the source position, except for PG $2214+139$ where the position is near the edge of the FIRST survey area.

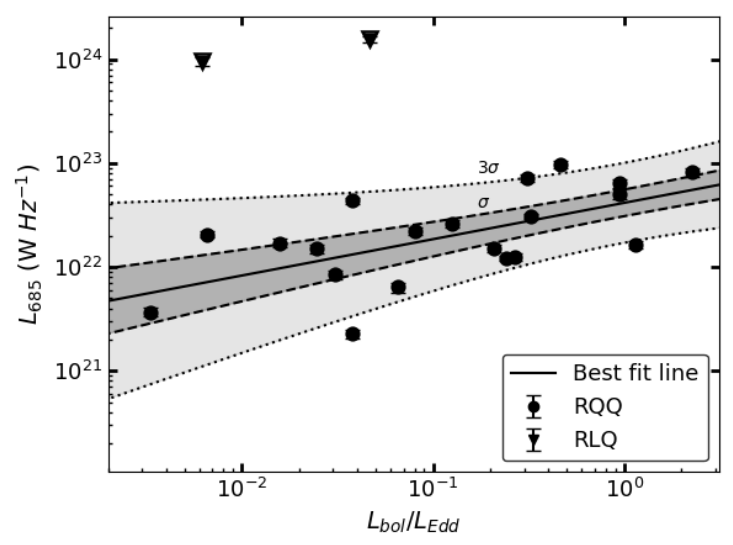

Figure 8. $685 \mathrm{MHz}$ k-corrected rest-frame luminosity as a function of Eddington ratio for the PG sources. The black solid line is the best-fitting line for the data. The black dashed lines mark the $1 \sigma$ regions and the black dotted lines mark the $3 \sigma$ regions.

Survey and PG survey, Lacy et al. (2001) found evidence for a strong dependence of $5 \mathrm{GHz}$ rest-frame luminosity on $\mathrm{BH}$ mass $\left(\mathrm{M}_{\mathrm{BH}}\right)$ and Eddington ratio $\left(\mathrm{L}_{\mathrm{bol}} / \mathrm{L}_{\mathrm{Edd}}\right)$. Using the $5 \mathrm{GHz}$ and $8.4 \mathrm{GHz}$ VLA data, Laor et al. (2019) observed a highly significant anticorrelation between $\alpha_{R}$ and $\mathrm{L}_{\text {bol }} / \mathrm{L}_{\mathrm{Edd}}$ and a marginal correlation between $\alpha_{R}$ and $\mathrm{M}_{\mathrm{BH}}$ for a different sub-sample of the RQ PG sample. Using the uGMRT $685 \mathrm{MHz}$ data, we searched for correlations between the radio properties, such as $\mathrm{L}_{685}, \mathrm{q}_{\mathrm{IR}}$, radio core size and $\alpha_{R}$ and the $\mathrm{BH}$ properties, such as, $\mathrm{M}_{\mathrm{BH}}$ and $\mathrm{L}_{\mathrm{bol}} / \mathrm{L}_{\mathrm{Edd}}$ for the RQ sources in our sample. We find a significant $(\sim 3 \sigma)$ correlation between $\mathrm{L}_{685}$ and $\mathrm{L}_{\text {bol }} / \mathrm{L}_{\text {Edd }}$ with $r_{s}=0.611$ and $\mathrm{p}_{s}=0.004$. This is presented in Figure 8, where the best-fitting line was obtained using a PYTHON fitting procedure - CURVE_FIT which also returns the optimal values of slope and intercept along with errors. The $1 \sigma$ and $3 \sigma$ scatter in the correlation were determined using these errors, assuming that they reflect the intrinsic scatter of the data. Moreover, the Spearman rank correlation test reveals no correlation between other radio and $\mathrm{BH}$ properties since the test yields $\mathrm{p}_{s}>$ $0.05 . \mathrm{M}_{\mathrm{BH}}$ and $\mathrm{AGN}$ bolometric luminosity values are taken from Shangguan et al. (2018) and Lyu et al. (2017) respectively. $\mathrm{M}_{\mathrm{BH}}$ are the single-epoch virial $\mathrm{BH}$ masses calculated using equation 4 of Ho \& $\mathrm{Kim}(2015)$ and $\mathrm{L}_{\text {bol }}=5.29 \mathrm{~L}_{\mathrm{IR}, \mathrm{AGN}}$ where $\mathrm{L}_{\mathrm{IR}, \mathrm{AGN}}$ is the AGN IR luminosity from Lyu et al. (2017). $\mathrm{L}_{\mathrm{Edd}}$ is calculated as $\mathrm{L}_{\mathrm{Edd}}\left(\mathrm{L}_{\odot}\right)=3.2 \times 10^{4}\left(\mathrm{M}_{\mathrm{BH}} / \mathrm{M}_{\odot}\right)$.

The lack of strong correlations between the $685 \mathrm{MHz}$ radio and $\mathrm{BH}$ properties (except for the one between $\mathrm{L}_{685}$ and $\mathrm{L}_{\text {bol }} / \mathrm{L}_{\mathrm{Edd}}$ ) in the RQ sources could be explained by the contribution of stellar-related emission, or radio emission from previous AGN activity episodes that may not be directly related to the current $\mathrm{BH}$ activity state. The lack of correlations may also suggest additional contributions from $\mathrm{BH}$ spin, magnetic flux, and environment.

\subsubsection{Coronal emission with uGMRT Data}

The emission from a magnetized corona likely located above the accretion disc was studied as a possible radio emission mechanism in RQ quasars by Laor \& Behar (2008) using the complete PG quasar sample. They observed a strong correlation between $5 \mathrm{GHz}$ radio luminosity (Kellermann et al. 1989, 1994) and 0.2-20 keV X-ray luminosity (Brandt et al. 2000; Laor \& Brandt 2002): $\mathrm{L}_{\mathrm{R}}\left(\mathrm{erg} \mathrm{s}^{-1}\right.$ ) 
$10^{-5} \mathrm{~L}_{\mathrm{X}}\left(\mathrm{erg} \mathrm{s}^{-1}\right)$, which is similar to the Guedel-Benz relation obeyed in coronally active stars for non-flaring radio and X-ray luminosity (Guedel \& Benz 1993):

$\log \mathrm{L}_{\mathrm{X}}\left(\mathrm{erg} \mathrm{s}^{-1}\right) \sim \log \mathrm{L}_{\mathrm{R}}\left(\mathrm{erg} \mathrm{s}^{-1} \mathrm{~Hz}^{-1}\right)+15.5$

The Guedel-Benz relation, along with correlated stellar X-ray and radio variability, indicates that the stellar corona is magnetically heated. Since RQ quasars and coronally active stars followed a similar $L_{R}-L_{X}$ relation, Laor \& Behar (2008) suggested that similar physical processes occurred in both objects. They proposed that the AGN corona is also magnetically heated and that both radio and $\mathrm{X}$-ray emission could originate from a compact corona.

The flux density $\left(\mathrm{S}_{5000}\right)$ and the k-corrected rest-frame luminosity at $5 \mathrm{GHz}\left(\mathrm{L}_{5000}\right)$ for the RQ sources in our PG sample are derived from the $685 \mathrm{MHz}$ uGMRT flux densities and $\alpha_{R}$ using equations 2 and 3 by substituting $v=5000 \mathrm{MHz}$. Figure 9 shows $\mathrm{L}_{X}$ as a function of $\mathrm{L}_{5000}$ for our RQ PG sources and compares them with the Guedel-Benz relation (equation 5). $\mathrm{L}_{X}$ values for individual sources are taken from Laor \& Behar (2008). It can be seen that the sources are not evenly scattered across the correlation but are biased towards a negative residual, unlike that observed in Laor \& Behar (2008). This could suggest that the coronal emission is not dominant at $685 \mathrm{MHz}$. This could also suggest that the $685 \mathrm{MHz}-$ $5 \mathrm{GHz}$ spectral index is flat for coronal emission. This would be consistent with the work of Raginski \& Laor (2016).

\section{CONCLUSIONS}

We have presented the first uGMRT $685 \mathrm{MHz}$ images of a subsample of 22 quasars belonging to the PG quasar sample. Two are radio-loud and 20 are radio-quiet.

(i) Nearly $80 \%$ sources are only a combination of a radio core unresolved at the uGMRT resolution of $\sim 3-5^{\prime \prime}$ surrounded by diffuse emission on few kpc to $\sim 10 \mathrm{kpc}$ scales and only four sources show extended radio emission on $\sim 10-30 \mathrm{kpc}$ scales.

(ii) The RL quasar cores have an inverted radio spectrum whereas the RQ quasar cores exhibit a range of spectral indices from flat to steep $\left(-0.1 \gtrsim \alpha_{R} \gtrsim-1.1\right)$. A few of these sources show signatures of unresolved/barely resolved jet/lobe structures in their spectral index images which are created using the $685 \mathrm{MHz}$ uGMRT data and similar resolution GHz-frequency VLA data.

(iii) On the basis of their position on the radio-IR correlation as well as the spectral index images, we find that the radio emission in the two RL quasars and nearly one-third of the RQ quasars is AGN dominated while the remaining sources appear to have significant contributions from both stellar-related processes and the AGN. Also, on the basis of their positional offset from the Guedel-Benz relation, it appears that the radio emission in the RQ PG sources at $685 \mathrm{MHz}$ does not have significant contribution from the magnetized coronal emission.

(iv) We searched for signatures of 'relic' emission in the PG sources. We find clear indications in one source, PG 0007+106, and suggestions of the same in a few others based on their spectral index images.

(v) We do not find strong correlations between the $685 \mathrm{MHz}$ radio properties, such as $\mathrm{L}_{685}$, $\mathrm{q}_{\mathrm{IR}}$, radio core size, and $\alpha_{R}$ and the $\mathrm{BH}$ properties, such as $\mathrm{M}_{\mathrm{BH}}$ and $\mathrm{L}_{\text {bol }} / \mathrm{L}_{\mathrm{Edd}}$ in the RQ sources except for the one between $\mathrm{L}_{685}$ and $\mathrm{L}_{\mathrm{bol}} / \mathrm{L}_{\mathrm{Edd}}$. This lack of correlations could be explained by the contribution of stellar-related emission, or radio emission from previous AGN activity episodes that may not be directly related to the current $\mathrm{BH}$ activity state.

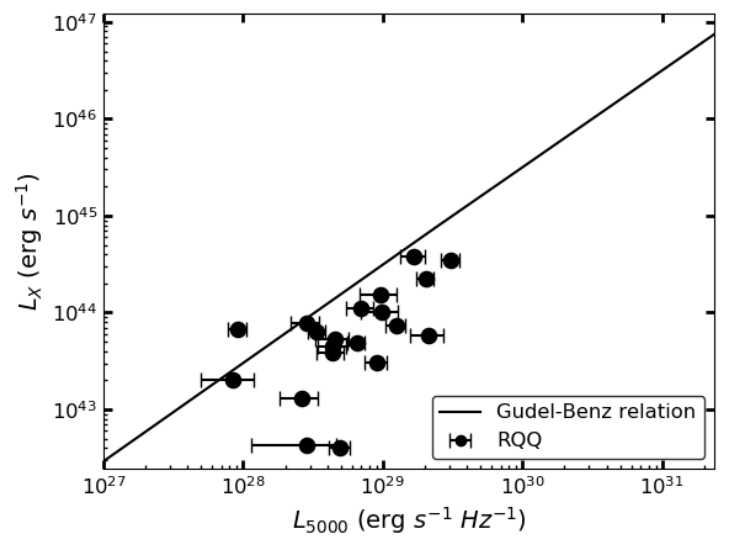

Figure 9. The 0.2-20 keV X-ray luminosity taken from Laor \& Behar (2008) as a function of $5000 \mathrm{MHz}$ k-corrected rest-frame luminosity for the RQ PG sources. The latter values are derived from $685 \mathrm{MHz}$ uGMRT flux densities and $\alpha_{R}$ using equations 2 and 3 by substituting $\nu=5000 \mathrm{MHz}$. The black solid line marks the Guedel-Benz relation obeyed in coronally active stars (Guedel \& Benz 1993).

The lack of correlations may also suggest additional contributions from other physical parameters, such as BH spin, magnetic flux, and environment.

\section{ACKNOWLEDGEMENTS}

We thank the referee for their valuable comments. $\mathrm{LCH}$ was supported by the National Science Foundation of China (11721303, 11991052) and the National Key R\&D Program of China (2016YFA0400702). We thank the staff of the GMRT that made these observations possible. GMRT is run by the National Centre for Radio Astrophysics of the Tata Institute of Fundamental Research. We acknowledge the support of the Department of Atomic Energy, Government of India, under the project 12-R\&D-TFR-5.020700. This research has made use of the NASA/IPAC Extragalactic Database (NED), which is funded by the National Aeronautics and Space Administration and operated by the California Institute of Technology.

\section{DATA AVAILABILITY}

The data underlying this article will be shared on reasonable request to the corresponding author. The raw data can be obtained from the GMRT Data Archive: https://naps.ncra.tifr.res.in/goa/data/search

\section{REFERENCES}

Ballard K. R., Heavens A. F., 1992, MNRAS, 259, 89 Barthel P. D., Arnaud K. A., 1996, MNRAS, 283, L45

Barvainis R., Lehár J., Birkinshaw M., Falcke H., Blundell K. M., 2005, ApJ, 618, 108

Begelman M. C., McKee C. F., Shields G. A., 1983, ApJ, 271, 70

Bell E. F., 2003, ApJ, 586, 794

Blandford R. D., Payne D. G., 1982, MNRAS, 199, 883

Blandford R. D., Rees M. J., 1974, MNRAS, 169, 395

Blandford R. D., Znajek R. L., 1977, MNRAS, 179, 433

Blundell K. M., Kuncic Z., 2007, ApJ, 668, L103 
Boroson T. A., Green R. F., 1992, ApJS, 80, 109

Brandt W. N., Laor A., Wills B. J., 2000, ApJ, 528, 637

Brunthaler A., Falcke H., Bower G. C., Aller M. F., Aller H. D., Teräsranta H., 2005, A\&A, 435, 497

Cappellari M., et al., 2013, MNRAS, 432, 1709

Carilli C. L., Perley R. A., Dhawan V., Perley D. A., 2019, ApJ, 874, L32

Cecil G., Bland-Hawthorn J., Veilleux S., Filippenko A. V., 2001, ApJ, 555, 338

Chiaraluce E., Panessa F., Bruni G., Baldi R. D., Behar E., Vagnetti F., Tombesi F., McHardy I., 2020, MNRAS, 495, 3943

Condon J. J., 1992, ARA\&A, 30, 575

Condon J. J., Cotton W. D., Broderick J. J., 2002, AJ, 124, 675

Condon J. J., Kellermann K. I., Kimball A. E., Ivezić Ž., Perley R. A., 2013, ApJ, 768, 37

Crenshaw D. M., Kraemer S. B., Gabel J. R., 2003, AJ, 126, 1690

Deo R. P., Crenshaw D. M., Kraemer S. B., 2006, AJ, 132, 321

Dunlop J. S., McLure R. J., Kukula M. J., Baum S. A., O’Dea C. P., Hughes D. H., 2003, MNRAS, 340, 1095

Evans A. S., Solomon P. M., Tacconi L. J., Vavilkin T., Downes D., 2006, AJ, 132, 2398

Everett J. E., 2005, ApJ, 631, 689

Falcke H., Nagar N. M., Wilson A. S., Ulvestad J. S., 2000, ApJ, 542, 197

Fanti C., Ficarra A., Gregorini L., Mantovani F., Olori M. C., 1981, A\&A, 97,251

Flewelling H. A., et al., 2016, arXiv e-prints, p. arXiv:1612.05243

Fukumura K., Kazanas D., Contopoulos I., Behar E., 2010, ApJ, 715, 636

Gallimore J. F., Axon D. J., O’Dea C. P., Baum S. A., Pedlar A., 2006, AJ, 132,546

Green R. F., Schmidt M., Liebert J., 1986, ApJS, 61, 305

Guedel M., Benz A. O., 1993, ApJ, 405, L63

Hardcastle M. J., et al., 2019, MNRAS, 488, 3416

Helou G., Soifer B. T., Rowan-Robinson M., 1985, ApJ, 298, L7

Ho L. C., 1999, ApJ, 510, 631

Ho L. C., 2002, ApJ, 564, 120

Ho L. C., Kim M., 2015, ApJ, 809, 123

Hota A., Saikia D. J., 2006, MNRAS, 371, 945

Hughes P. A., Aller H. D., Aller M. F., 1992, ApJ, 396, 469

Hutchings J. B., Crampton D., 1990, AJ, 99, 37

Hutchings J. B., Neff S. G., 1992, AJ, 104, 1

Hwang H.-C., Zakamska N. L., Alexand roff R. M., Hamann F., Greene J. E., Perrotta S., Richards G. T., 2018, MNRAS, 477, 830

Intema H. T., 2014, in Astronomical Society of India Conference Series. p. 469 (arXiv: 1402.4889 )

Irwin J. A., Saikia D. J., 2003, MNRAS, 346, 977

Jahnke K., Kuhlbrodt B., Wisotzki L., 2004, MNRAS, 352, 399

Jarvis M. E., et al., 2019, MNRAS, 485, 2710

Kaspi S., Smith P. S., Netzer H., Maoz D., Jannuzi B. T., Giveon U., 2000, ApJ, 533, 631

Kellermann K. I., Sramek R., Schmidt M., Shaffer D. B., Green R., 1989, AJ, 98, 1195

Kellermann K. I., Sramek R. A., Schmidt M., Green R. F., Shaffer D. B., 1994, AJ, 108, 1163

Kellermann K. I., Condon J. J., Kimball A. E., Perley R. A., Ivezić Ž., 2016, ApJ, 831, 168

Kennicutt Robert C. J., 1998, ARA\&A, 36, 189

Kharb P., Lister M. L., Marshall H. L., Hogan B. S., 2012, ApJ, 748, 81

Kharb P., Srivastava S., Singh V., Gallimore J. F., Ishwara-Chandra C. H., Ananda H., 2016, MNRAS, 459, 1310

Kharb P., Vaddi S., Sebastian B., Subramanian S., Das M., Paragi Z., 2019, ApJ, 871, 249

Kim M., Ho L. C., Peng C. Y., Barth A. J., Im M., Martini P., Nelson C. H., 2008, ApJ, 687, 767

Kim M., Ho L. C., Peng C. Y., Barth A. J., Im M., 2017, ApJS, 232, 21

Konigl A., Kartje J. F., 1994, ApJ, 434, 446

Kratzer R. M., Richards G. T., 2015, AJ, 149, 61

La Franca F., Melini G., Fiore F., 2010, ApJ, 718, 368

Lacy M., Laurent-Muehleisen S. A., Ridgway S. E., Becker R. H., White R. L., 2001, ApJ, 551, L17
Lal D. V., Ho L. C., 2010, AJ, 139, 1089

Laor A., Behar E., 2008, MNRAS, 390, 847

Laor A., Brandt W. N., 2002, ApJ, 569, 641

Laor A., Baldi R. D., Behar E., 2019, MNRAS, 482, 5513

Lyu J., Rieke G. H., Shi Y., 2017, ApJ, 835, 257

McLure R. J., Jarvis M. J., 2004, MNRAS, 353, L45

Mizumoto M., Done C., Tomaru R., Edwards I., 2019, MNRAS, 489, 1152

Morić I., Smolčić V., Kimball A., Riechers D. A., Ivezić Ž., Scoville N., 2010, ApJ, 724, 779

Nims J., Quataert E., Faucher-Giguère C.-A., 2015, MNRAS, 447, 3612

O’Dea C. P., Baum S. A., Stanghellini C., 1991, ApJ, 380, 66

Ohta K., Aoki K., Kawaguchi T., Kiuchi G., 2007, ApJS, 169, 1

Orienti M., 2016, Astronomische Nachrichten, 337, 9

Panessa F., Baldi R. D., Laor A., Padovani P., Behar E., McHardy I., 2019, Nature Astronomy, 3, 387

Petric A. O., Ho L. C., Flagey N. J. M., Scoville N. Z., 2015, ApJS, 219, 22

Proga D., Kallman T. R., 2004, ApJ, 616, 688

Raginski I., Laor A., 2016, MNRAS, 459, 2082

Rees M. J., 1984, ARA\&A, 22, 471

Roettiger K., Burns J. O., Clarke D. A., Christiansen W. A., 1994, ApJ, 421, L23

Schinnerer E., Eckart A., Tacconi L. J., 1998, ApJ, 500, 147

Shang Z., et al., 2011, ApJS, 196, 2

Shangguan J., Ho L. C., Xie Y., 2018, ApJ, 854, 158

Shangguan J., Ho L. C., Bauer F. E., Wang R., Treister E., 2020, ApJS, 247 , 15

Shaw R. A., Hill F., Bell D. J., 2007, Astronomical Data Analysis Software and Systems XVI. Vol. 376

Shi Y., Rieke G. H., Ogle P. M., Su K. Y. L., Balog Z., 2014, ApJS, 214, 23

Sikora M., Stawarz Ł., Lasota J.-P., 2007, ApJ, 658, 815

Stannard D., Bentley M., 1977, MNRAS, 180, 703

Surace J. A., Sanders D. B., Evans A. S., 2001, AJ, 122, 2791

Tarenghi M., Garilli B., Maccagni D., 1994, AJ, 107, 1629

Tchekhovskoy A., Narayan R., McKinney J. C., 2010, ApJ, 711, 50

Terzian Y., 1965, ApJ, 142, 135

Torniainen I., Tornikoski M., Teräsranta H., Aller M. F., Aller H. D., 2005 , A\&A, 435, 839

Urry M., 2003, The AGN Paradigm for Radio-Loud Objects. p. 3

Veilleux S., et al., 2009a, ApJS, 182, 628

Veilleux S., et al., 2009b, ApJ, 701, 587

Vestergaard M., Peterson B. M., 2006, ApJ, 641, 689

Wells D. C., 1985, in di Gesu V., Scarsi L., Crane P., Friedman J. H., Levialdi S., eds, Data Analysis in Astronomy. p. 195

White R. L., et al., 2000, ApJS, 126, 133

Wilkinson P. N., Polatidis A. G., Readhead A. C. S., Xu W., Pearson T. J., 1994, ApJ, 432, L87

Wilson A. S., Colbert E. J. M., 1995, ApJ, 438, 62

Wong O. I., et al., 2016, MNRAS, 460, 1588

Woods D. T., Klein R. I., Castor J. I., McKee C. F., Bell J. B., 1996, ApJ, 461, 767

Yun M. S., Reddy N. A., Condon J. J., 2001, ApJ, 554, 803

Zakamska N. L., Greene J. E., 2014, MNRAS, 442, 784

Zakamska N. L., et al., 2016, MNRAS, 455, 4191

Zheng Z., Wu H., Mao S., Xia X. Y., Deng Z. G., Zou Z. L., 1999, A\&A, 349, 735

Zhuang M.-Y., Ho L. C., Shangguan J., 2018, ApJ, 862, 118

Zwicky F., Zwicky M. A., 1971, Catalogue of selected compact galaxies and of post-eruptive galaxies

de Vaucouleurs G., de Vaucouleurs A., Corwin Herold G. J., Buta R. J., Paturel G., Fouque P., 1991, Third Reference Catalogue of Bright Galaxies

\section{APPENDIX A: OPTICAL MORPHOLOGY}

Here, we discuss the optical morphology of the host galaxies.

PG 0003 + 199: The host could either be a lenticular galaxy or an unbarred spiral galaxy with tightly wound spiral arms (type S0/a; 
from NED). However, Crenshaw et al. (2003), Deo et al. (2006) and Ohta et al. (2007) classify this as a 'point source' (type P), meaning the nucleus dominates the light distribution. The PanSTARRS image reveals an optical jet-like feature emerging from the host galaxy that indicates that the galaxy is in some state of interaction. Ohta et al. (2007) also suggests that this is an interacting or a peculiar galaxy. The HST image analysis reveals the presence of a bulge and an extended disc (Kim et al. 2017).

PG 0007 + 106: This source is classified as an Optically Violent Variable (OVV) quasar (Veilleux et al. 2009b). The disturbedlooking host galaxy is part of an interconnected triplet of compact galaxies (Zwicky \& Zwicky 1971).

PG $0050+124$ : The host is a starburst unbarred spiral galaxy with tightly wound spiral arms (type Sa;Sbrst; from NED, see also Hutchings \& Crampton 1990; Schinnerer et al. 1998). The two asymmetric spiral arms have knots of SF (Surace et al. 2001). These tidal tails could be a result of merging (Zheng et al. 1999).

PG 0923 + 129: The host is classified as a lenticular galaxy, marked with an uncertainity in NED (type S0?; de Vaucouleurs et al. 1991). However, Ohta et al. (2007) classifies the host as a lenticular galaxy with an outer ring (type RS0). The presence of the outer ring is also evident from the PanSTARRS optical image (see Figure B2 in APPENDIX B). One of the scenarios suggested is that the ring galaxies are formed when a smaller galaxy passes through the center of a larger galaxy. The radio source in the south is associated with a neighbouring host galaxy, which could be the merger companion in the ring formation.

PG 0934 + 013: The host is a barred spiral galaxy with weakly wound spiral arms (type SBab; from NED and Ohta et al. 2007).

PG 1011 - 010: The host is a barred spiral galaxy with loosely wound spiral arms and possessing inner ring-like structures and morphological peculiarities (type SB(r)b: pec; from NED and Ohta et al. 2007).

PG 1119 + 120: The host galaxy is an intermediate spiral galaxy with tightly wound spiral arms (type SABa; from NED and Ohta et al. 2007).

PG 1211 + 143: The HST image analysis suggests the presence of a faint central feature resembling a bar or highly inclined disclike structure. However, it is not clear if this is an artefact due to point-spread function mismatch (Kim et al. 2008).

PG 1229 + 204: The host is a barred lenticular galaxy (type SB0; from NED). However, (Surace et al. 2001) classifies the host as a barred spiral galaxy (type SB). An asymmetric nuclear bar runs in the NE-SW direction through the host (Surace et al. 2001). The northern arm of the bar is wider than the southern arm. This luminous extension on one side of the bar is thought to be due to a small merging companion (Hutchings \& Neff 1992). The HST image analysis reveals the presence of a faint tidal tail, which may be signature of interaction or minor merger (Kim et al. 2017).

PG 1244 + 026: The host could either be an elliptical galaxy or a lenticular galaxy (type E/S0; from NED and Ohta et al. 2007).

PG 1310 - 108: Jahnke et al. (2004) finds a clear disc with two asymmetric spiral or tidal arms. No luminous companion is visible as a source of tidal interaction; the closest ones being two galaxies with unknown redshifts, one which is 0.5 mag fainter than the host in $\mathrm{V}$ and $45 \mathrm{kpc}$ to the north, and the other one is 2 mag fainter and $110 \mathrm{kpc}$ east. We expect a case of minor merger here (Sikora et al. 2007).

PG 1426 + 015: The host could either be an elliptical galaxy or a lenticular galaxy (type E/S0; from NED). The HST image analysis reveals a tidal tail and a small companion (Kim et al. 2008) and also suggests that it is a disturbed elliptical galaxy (Kim et al. 2017).
PG 1448 + 273: The PanSTARRS image of the host galaxy reveals the presence of tidal tails. Ohta et al. (2007) also suggests that this is an interacting or a peculiar galaxy.

PG 1501 + 106: The host is an elliptical galaxy (type E; from NED and Tarenghi et al. 1994).

PG 2130 + 099: The host is an unbarred spiral galaxy with tightly wound spiral arms and outer ring-like structures (type (R)Sa; from NED). The HST image analysis reveals a ring-like spiral disc (Kim et al. 2008) and also suggests the presence of a pseudo-bulge and a disturbed disc (Kim et al. 2017).

PG 2214 + 139: This source is classified as an Ultra Luminous Infrared Galaxy (ULIRG) by Veilleux et al. (2009a).

\section{APPENDIX B: SUPPLEMENTARY DATA}

We present the $685 \mathrm{MHz}$ uGMRT images, radio-optical overlays and radio spectral index images in this section.

This paper has been typeset from a $\mathrm{T}_{\mathrm{E}} \mathrm{X} / \mathrm{L} \mathrm{A} \mathrm{T}_{\mathrm{E}} \mathrm{X}$ file prepared by the author. 

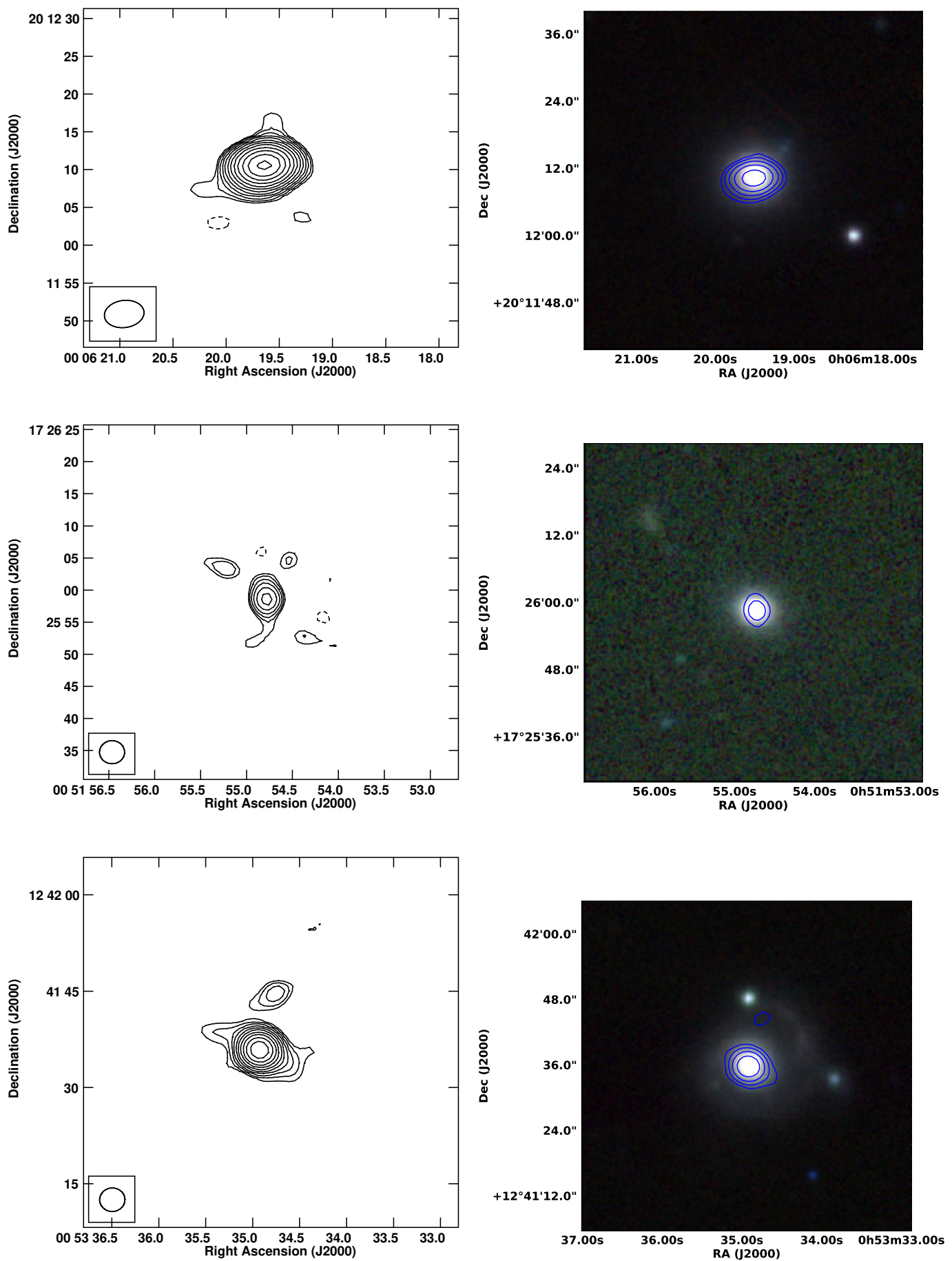

Figure B1. Left: $685 \mathrm{MHz}$ total intensity contour images of PG 0003+199, PG 0049+171 and PG 0050+124 from top to bottom. The peak contour flux is $x$ $\mathrm{mJy}_{\text {beam }}{ }^{-1}$ and the contour levels are $y \times(-1,1,1.4,2,2.8,4,5.6,8,11.20,16,23,32,45,64,90,128,180,256,362,512) \mathrm{mJy} \mathrm{beam}^{-1}$, where $(x ; y)$ for PG 0003+199, PG 0049+171 and PG 0050+124 are (144;0.23), (1055.7;0.28) and (112.6;0.30) respectively. Right: 685 MHz total intensity contours in blue superimposed on PanSTARRS griz-color composite optical images of PG 0003+199, PG 0049+171 and PG 0050+124 from top to bottom. The peak contour flux is $x$ mJy beam $^{-1}$ and the contour levels are $y \times(2,4,8,16,32,64,128,256,512)$ mJy beam ${ }^{-1}$, where $(x ; y)$ for PG 0003+199, PG 0049+171 and PG $0050+124$ are (144;0.23), (1055.7;0.28) and (112.6;0.30) respectively. 

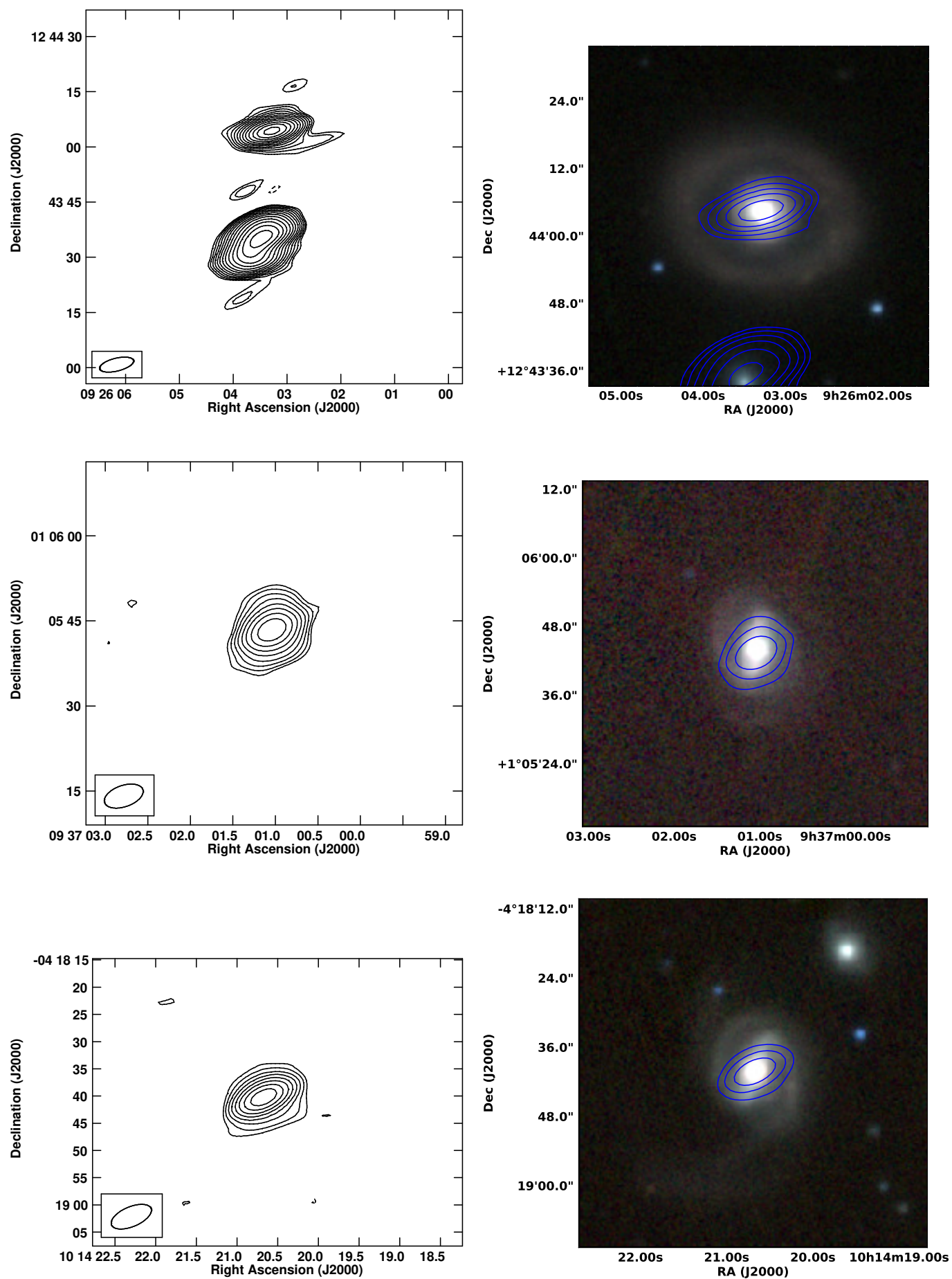

Figure B2. Left: $685 \mathrm{MHz}$ total intensity contour images of PG 0923+129, PG 0934+013 and PG 1011-040 from top to bottom. The peak contour flux is $x \mathrm{mJy}_{\text {beam }^{-1}}$ and the contour levels are $y \times(-1,1,1.4,2,2.8,4,5.6,8,11.20,16,23,32,45,64,90,128,180,256,362,512) \mathrm{mJy}_{\text {beam }}^{-1}$, where $(x ; y)$ for PG $0923+129$, PG $0934+013$ and PG 1011-040 are (30;0.22), (35.9;0.08) and (42.3;0.08) respectively. Right: $685 \mathrm{MHz}$ total intensity contours in blue superimposed on PanSTARRS griz-color composite optical image of PG 0923+129, PG 0934+013 and PG 1011-040 from top to bottom. The peak contour flux is $x$ mJy beam $^{-1}$ and the contour levels are $y \times(2,4,8,16,32,64,128,256,512) \mathrm{mJy}_{\text {beam }}{ }^{-1}$, where $(x ; y)$ for PG 0923+129, PG 0934+013 and PG $1011-040$ are $(30 ; 0.22),(35.9 ; 0.08)$ and $(42.3 ; 0.08)$ respectively. 

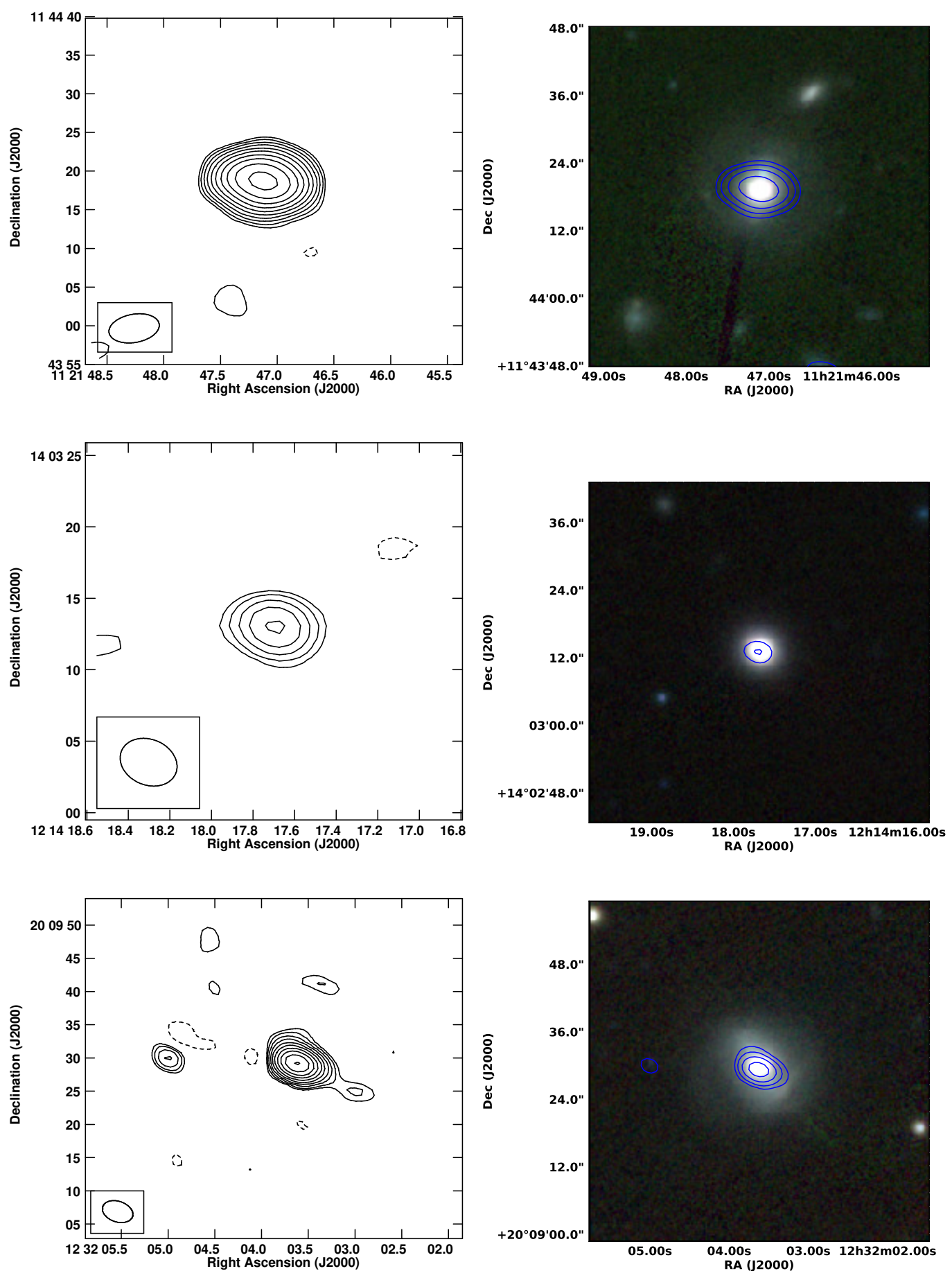

Figure B3. Left: $685 \mathrm{MHz}$ total intensity contour images of PG 1119+120, PG 1211+143 and PG 1229+204 from top to bottom. The peak contour flux is $x$ $\mathrm{mJy}_{\text {beam }}{ }^{-1}$ and the contour levels are $y \times(-1,1,1.4,2,2.8,4,5.6,8,11.20,16,23,32,45,64,90,128,180,256,362,512) \mathrm{mJy}$ beam ${ }^{-1}$, where $(x ; y)$ for PG 1119+120, PG 1211+143 and PG 1229+204 are (66.7;0.12), (1211.4;1.1) and (160.6;0.08) respectively. Right: 685 MHz total intensity contours in blue superimposed on PanSTARRS griz-color composite optical image of PG 1119+120, PG 1211+143 and PG 1229+204 from top to bottom. The peak contour flux is $x$ mJy beam $^{-1}$ and the contour levels are $y \times(2,4,8,16,32,64,128,256,512) \mathrm{mJy}_{\text {beam }}{ }^{-1}$, where $(x ; y)$ for PG $1119+120$, PG $1211+143$ and PG $1229+204$ are $(66.7 ; 0.12),(1211.4 ; 1.1)$ and $(160.6 ; 0.08)$ respectively. 

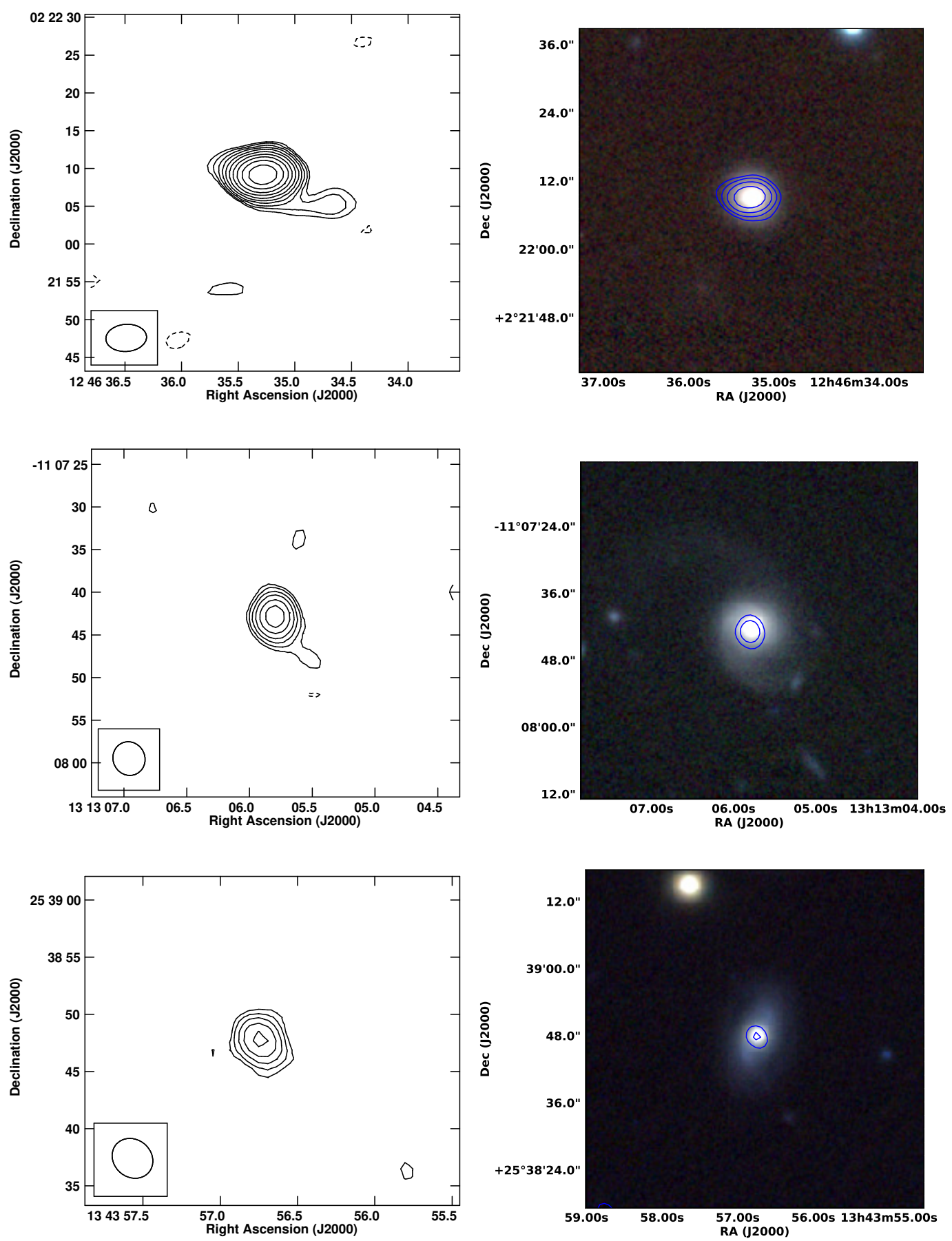

Figure B4. Left: $685 \mathrm{MHz}$ total intensity contour images of PG 1244+026, PG 1310-108 and PG 1341+258 from top to bottom. The peak contour flux is $x$ $\mathrm{mJy}_{\text {beam }}{ }^{-1}$ and the contour levels are $y \times(-1,1,1.4,2,2.8,4,5.6,8,11.20,16,23,32,45,64,90,128,180,256,362,512) \mathrm{mJy}$ beam ${ }^{-1}$, where $(x ; y)$ for PG 1244+026, PG 1310-108 and PG 1341+258 are (41.9;0.09), (51.67;0.10) and (95.69;0.07) respectively. Right: 685 MHz total intensity contours in blue superimposed on PanSTARRS griz-color composite optical image of PG 1244+026, PG 1310-108 and PG 1341+258 from top to bottom. The peak contour flux is $x \mathrm{mJy}_{\text {beam }}{ }^{-1}$ and the contour levels are $y \times(2,4,8,16,32,64,128,256,512) \mathrm{mJy}_{\text {beam }}{ }^{-1}$, where $(x ; y)$ for PG $1244+026$, PG $1310-108$ and PG $1341+258$ are $(41.9 ; 0.09),(51.67 ; 0.10)$ and $(95.69 ; 0.07)$ respectively. 

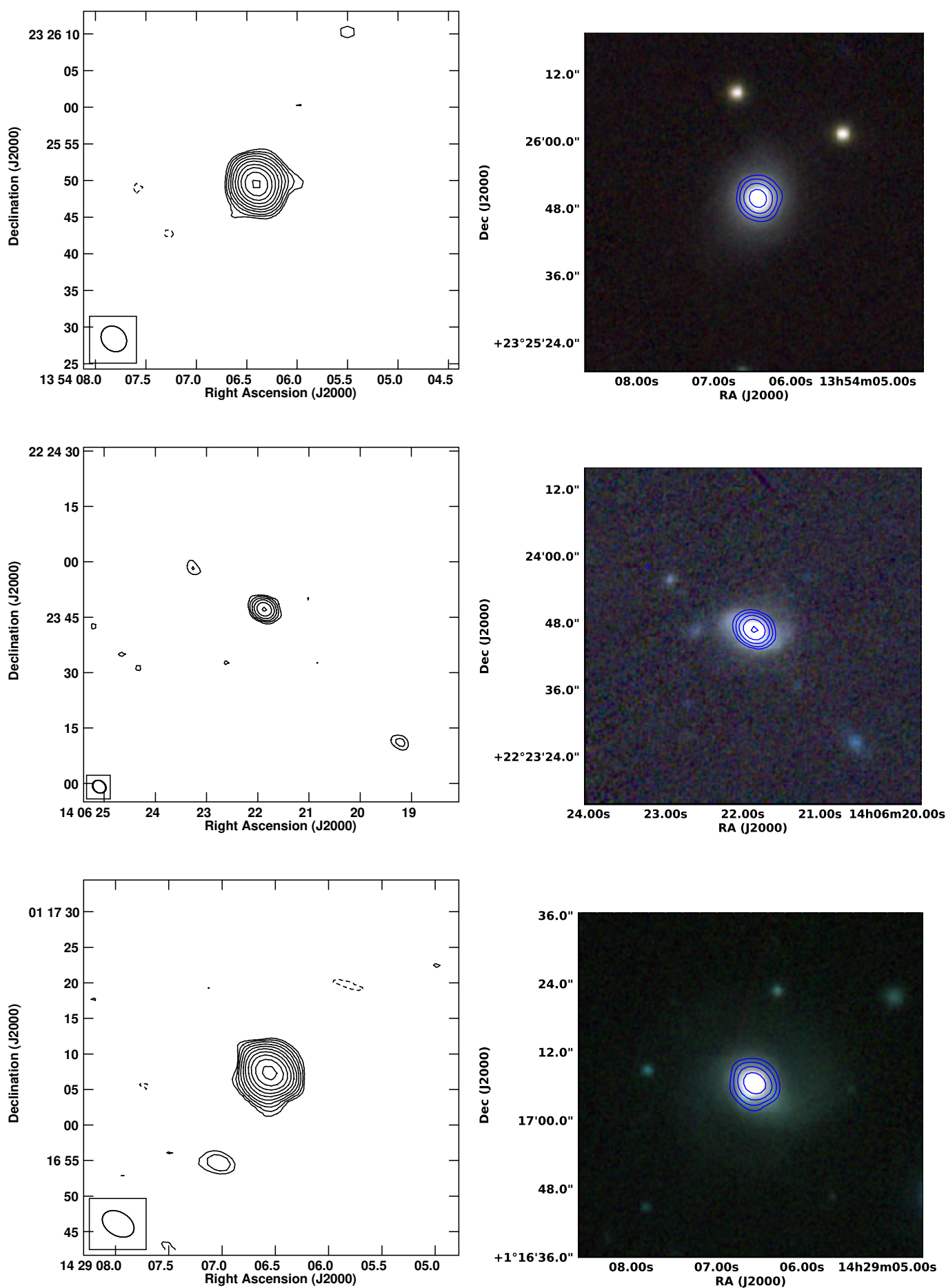

Figure B5. Left: $685 \mathrm{MHz}$ total intensity contour images of PG 1351+236, PG 1404+226 and PG 1426+015 from top to bottom. The peak contour flux is $x$ $\mathrm{mJy}_{\text {beam }}{ }^{-1}$ and the contour levels are $y \times(-1,1,1.4,2,2.8,4,5.6,8,11.20,16,23,32,45,64,90,128,180,256,362,512) \mathrm{mJy}_{\text {beam }}^{-1}$, where $(x ; y)$ for PG $1351+236$ and PG $1426+015$ are $(34.2 ; 0.08)$ and $(48.8 ; 0.08)$ respectively. The peak contour flux for PG $1404+226$ is $143.3 \mathrm{mJy}^{-1}$ beam ${ }^{-1}$ and the contour levels are $0.08 \times(-1,1,2,4,8,16,32,64,128,256,512) \mathrm{mJy} \mathrm{beam}^{-1}$. Right: $685 \mathrm{MHz}$ total intensity contours in blue superimposed on PanSTARRS griz-color composite optical image of PG 1351+236, PG 1404+226 and PG 1426+015 from top to bottom. The peak contour flux is $x \mathrm{mJy}^{-1}$ beam ${ }^{-1}$ and the contour levels are $y \times(2,4,8,16,32,64,128,256,512) \mathrm{mJy}_{\text {beam }}{ }^{-1}$, where $(x ; y)$ for PG 1351+236, PG 1404+226 and PG 1426+015 are (34.2;0.08), (143.3;0.08) and $(48.8 ; 0.08)$ respectively. 

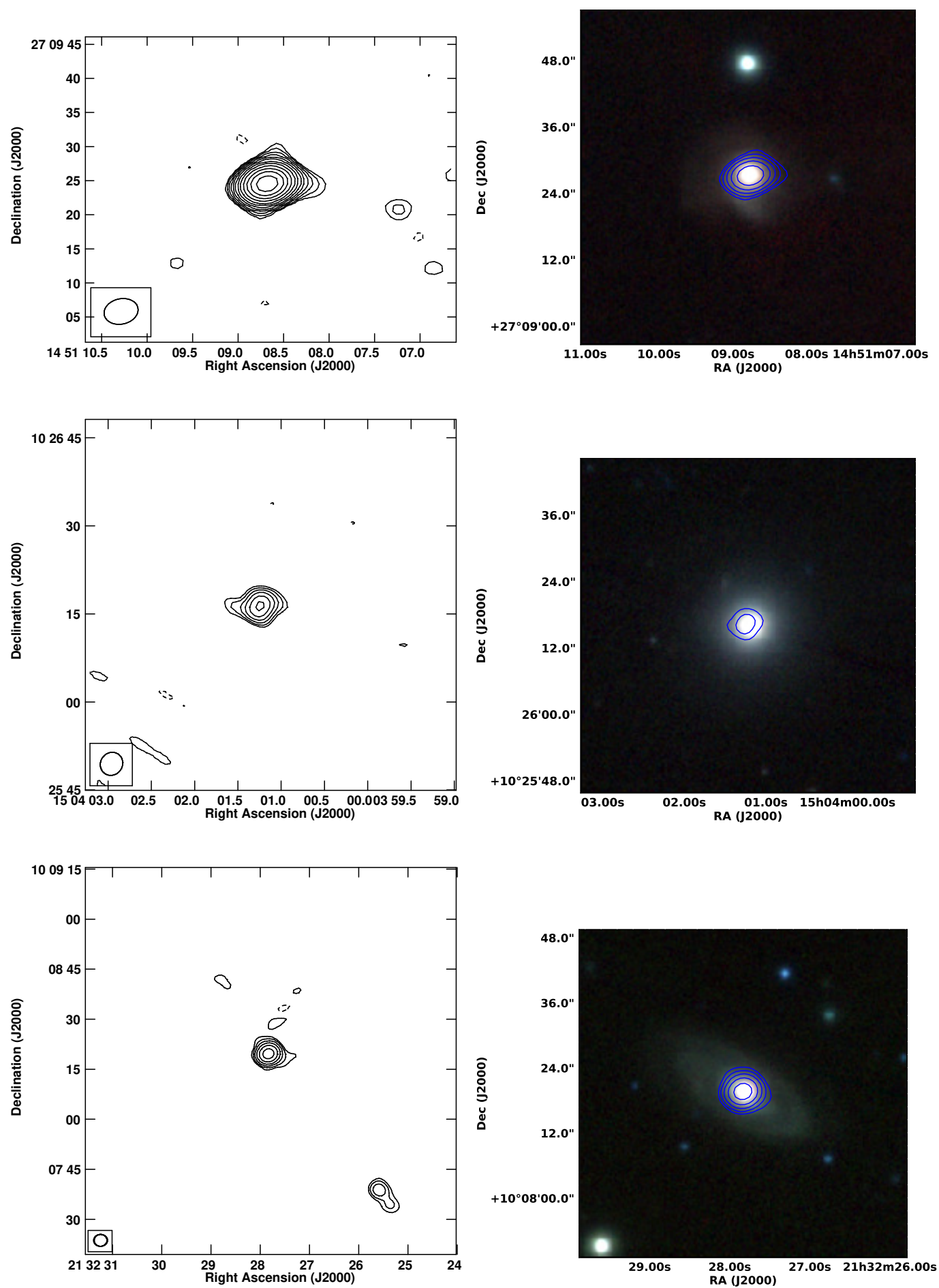

Figure B6. Left: $685 \mathrm{MHz}$ total intensity contour images of PG 1448+273, PG 1501+106 and PG 2130+099 from top to bottom. The peak contour flux is $x$ $\mathrm{mJy}_{\text {beam }}{ }^{-1}$ and the contour levels are $y \times(-1,1,1.4,2,2.8,4,5.6,8,11.20,16,23,32,45,64,90,128,180,256,362,512) \mathrm{mJy}_{\text {beam }}^{-1}$, where $(x ; y)$ for PG $1448+273$ and PG $1501+106$ are $(18.4 ; 0.09)$ and $(1469.5 ; 0.6)$ respectively. The peak contour flux for PG $2130+099$ is $67.2 \mathrm{mJy}^{-1}$ beam ${ }^{-1}$ and the contour levels are $0.15 \times(-1,1,2,4,8,16,32,64,128,256,512) \mathrm{mJy}_{\text {beam }}^{-1}$. Right: $685 \mathrm{MHz}$ total intensity contours in blue superimposed on PanSTARRS griz-color composite optical image of PG 1448+273, PG 1501+106 and PG 2130+099 from top to bottom. The peak contour flux is $x$ mJy beam $^{-1}$ and the contour levels are $y \times(2,4,8,16,32,64,128,256,512) \mathrm{mJy}$ beam $^{-1}$, where $(x ; y)$ for PG 1448+273, PG 1501+106 and PG 2130+099 are $(18.4 ; 0.09),(1469.5 ; 0.6)$ and $(67.2 ; 0.15)$ respectively. 

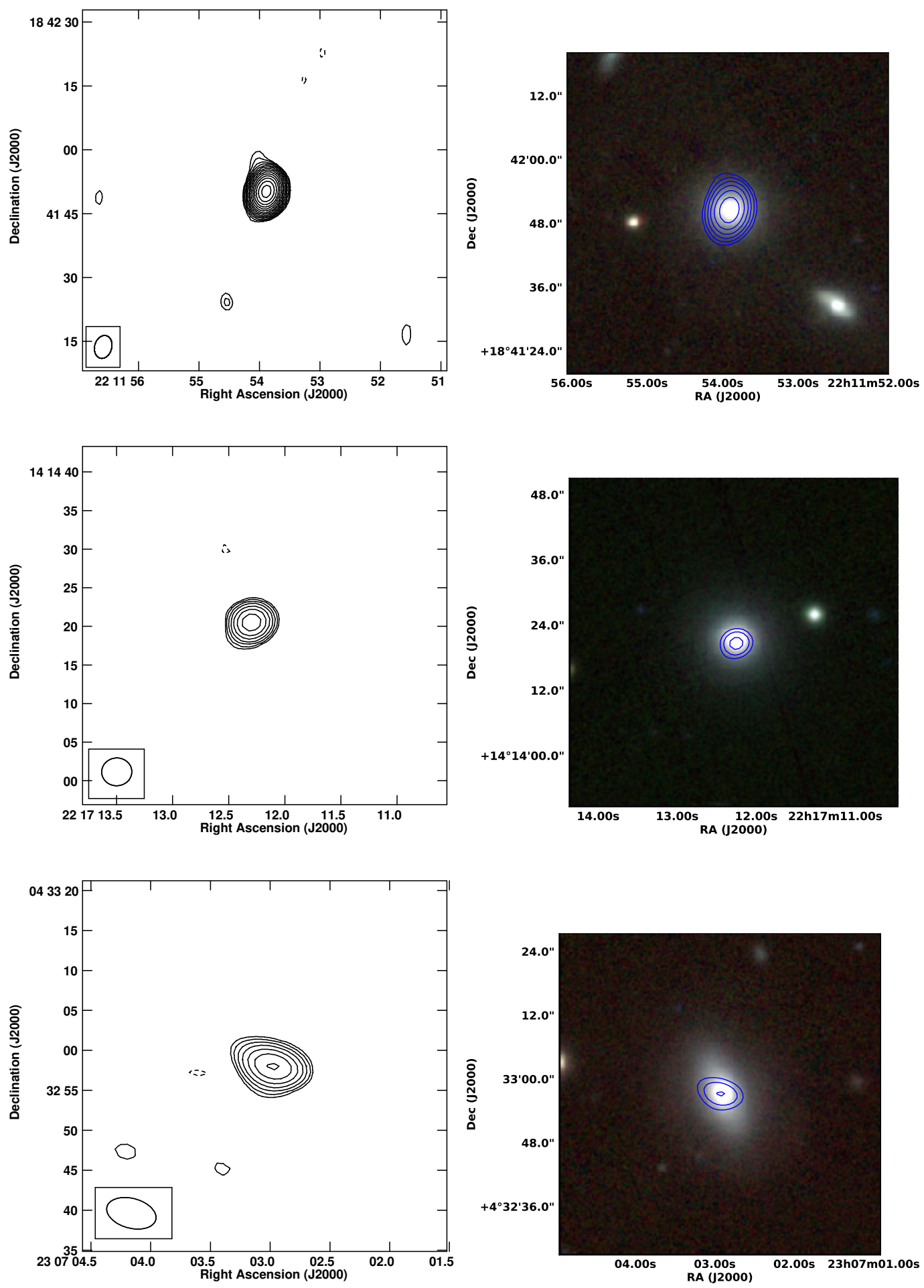

Figure B7. Left: $685 \mathrm{MHz}$ total intensity contour images of PG 2209+184, PG 2214+139 and PG 2304+042 from top to bottom. The peak contour flux is $x$ mJy beam ${ }^{-1}$ and the contour levels are $y \times(-1,1,1.4,2,2.8,4,5.6,8,11.20,16,23,32,45,64,90,128,180,256,362,512) \mathrm{mJy} \mathrm{beam}^{-1}$, where $(x ; y)$ for PG 2209+184, PG 2214+139 and PG 2304+042 are (333;0.85), (27.34;0.09) and (321.8;0.12) respectively. Right: $685 \mathrm{MHz}$ total intensity contours in blue superimposed on PanSTARRS griz-color composite optical image of PG 2209+184, PG 2214+139 and PG 2304+042 from top to bottom. The peak contour flux is $x$ mJy beam $^{-1}$ and the contour levels are $y \times(2,4,8,16,32,64,128,256,512) \mathrm{mJy}_{\text {beam }}{ }^{-1}$, where $(x ; y)$ for PG 2209+184, PG 2214+139 and PG $2304+042$ are (333;0.85), (27.34;0.09) and (321.8;0.12) respectively. 

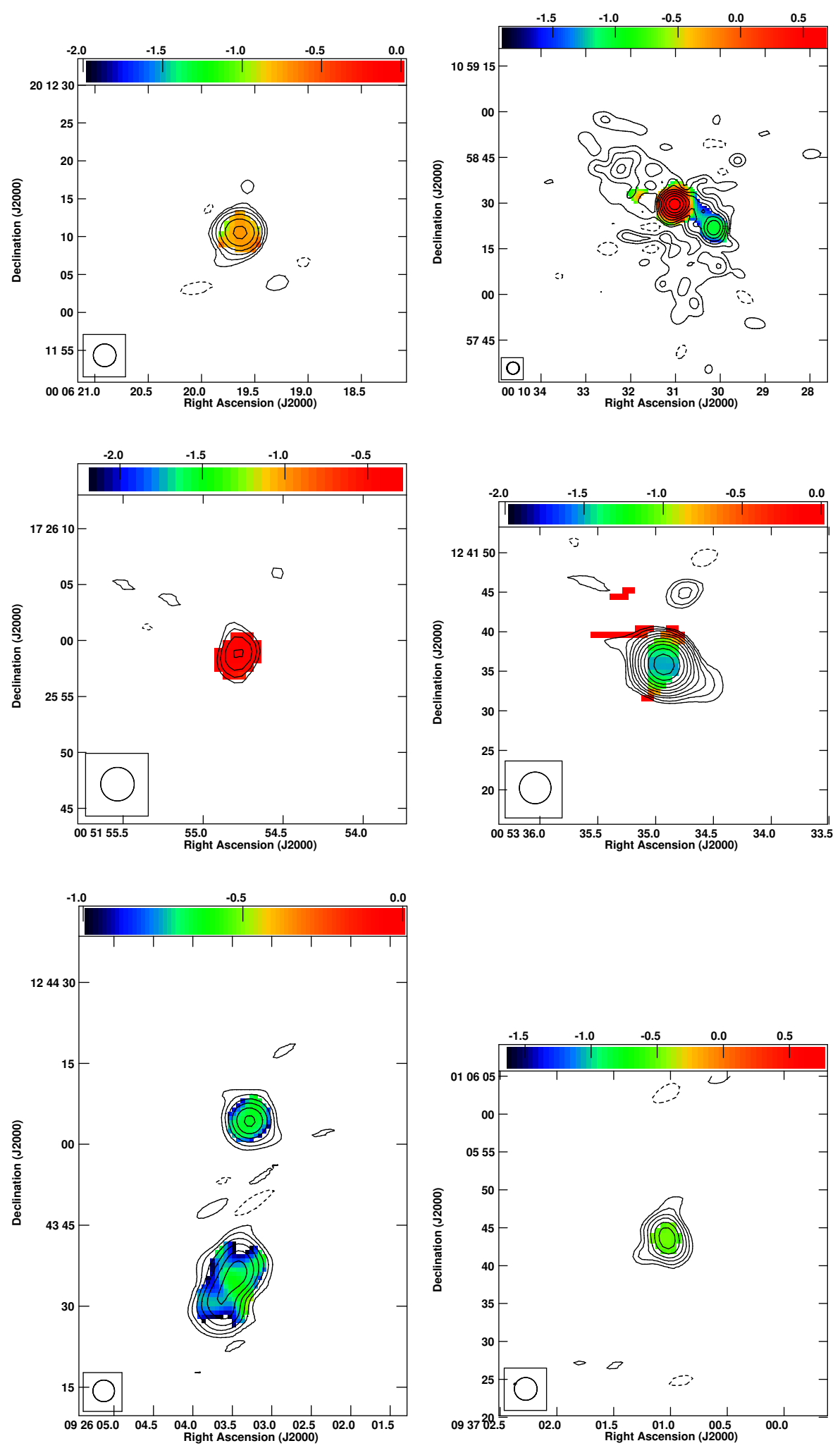

Figure B8. $685 \mathrm{MHz}$ total intensity contours in black superimposed on spectral index image in color for Top left: PG 0003+199; Top right: PG 0007+106; Middle left: PG 0049+171; Middle right: PG 0050+124; Bottom left: PG 0923+129; Bottom right: PG 0934+013. The peak contour flux is $x$ mJy beam $^{-1}$ and the contour levels are $y \times(-1,1,2,4,8,16,32,64,128,256,512) \mathrm{mJy} \mathrm{beam}^{-1}$, where $(x ; y)$ for PG 0003+199, PG 0007+106 and PG 0923+129 are $(135 ; 0.26),(54 ; 0.25)$ and $(27 ; 0.30)$ respectively. The peak contour flux is $x^{*} \mathrm{mJy} \mathrm{beam}^{-1}$ and the contour levels are $y^{*} \times(-1,1,1.4,2,2.8,4,5.6,8,11.20,16$, $23,32,45,64,90,128,180,256,362,512) \mathrm{mJy}$ beam ${ }^{-1}$, where $\left(x^{*} ; y^{*}\right)$ for PG $0049+171$, PG $0050+124$ and PG $0934+013$ are $(989.4 ; 0.59),(17.9 ; 0.32)$ and $(24.9 ; 0.12)$ respectively. 

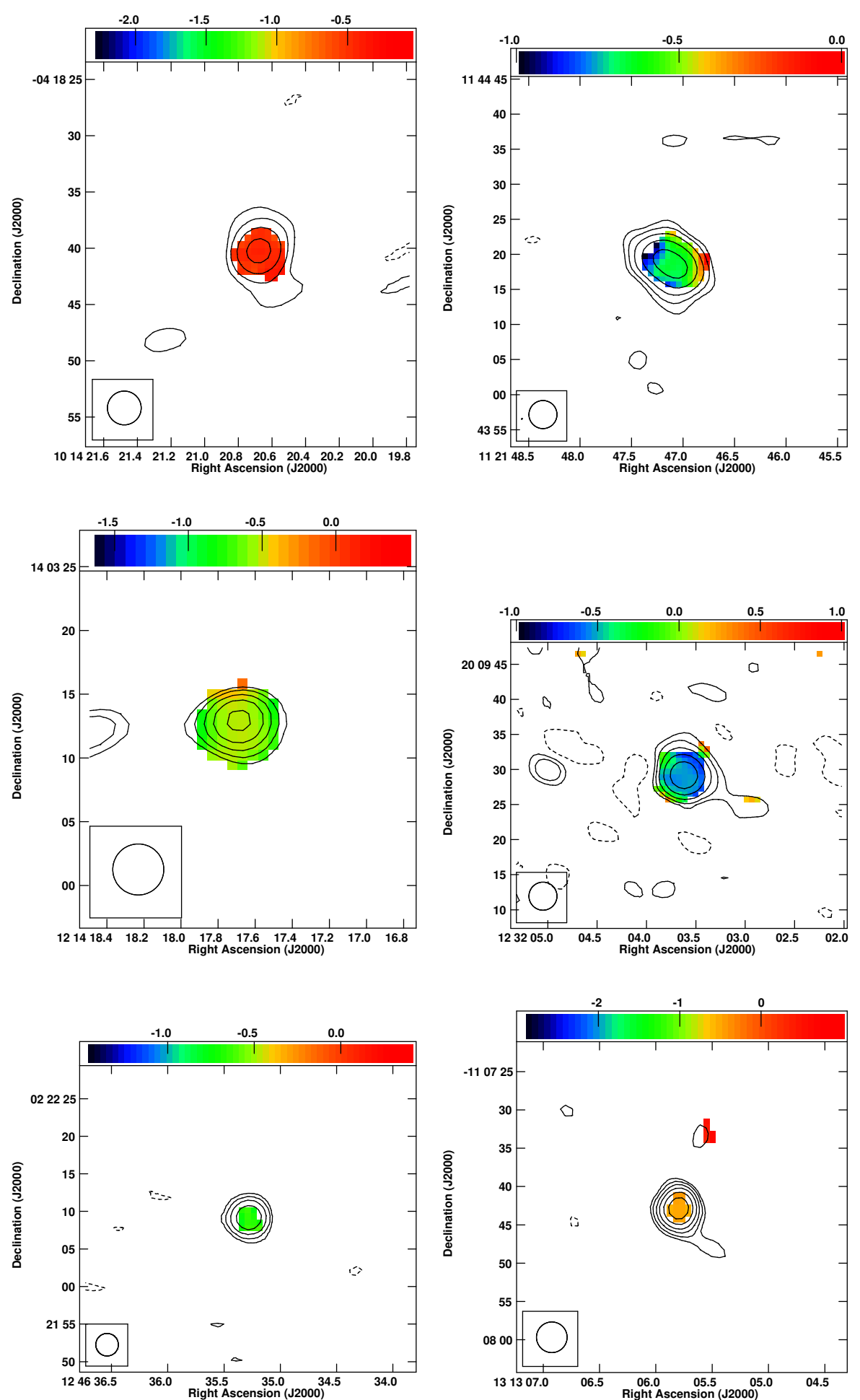

Figure B9. $685 \mathrm{MHz}$ total intensity contours in black superimposed on spectral index image in color for Top left: PG 1011-040; Top right: PG 1119+120; Middle left: PG 1211+143; Middle right: PG 1229+204; Bottom left: PG 1244+026; Bottom right: PG 1310-108. The peak contour flux is $x \mathrm{mJy}^{2}$ beam ${ }^{-1}$ and the contour levels are $y \times(-1,1,2,4,8,16,32,64,128,256,512) \mathrm{mJy}_{\text {beam }}{ }^{-1}$, where $(x ; y)$ for PG 1011-040, PG 1119+120, PG 1229+204 and PG $1244+026$ are $(40.8 ; 0.10),(64.6 ; 0.12),(166.1 ; 0.06)$ and $(39.1 ; 0.16)$ respectively. The peak contour flux is $x^{*}$ mJy beam ${ }^{-1}$ and the contour levels are $y * \times(-1,1,1.4,2$, $2.8,4,5.6,8,11.20,16,23,32,45,64,90,128,180,256,362,512) \mathrm{mJy} \mathrm{beam}^{-1}$, where $\left(x^{*} ; y^{*}\right)$ for PG $1211+143$ and PG $1310-108$ are $(1200.3 ; 1.0)$ and $(53.76 ; 0.10)$ respectively. 

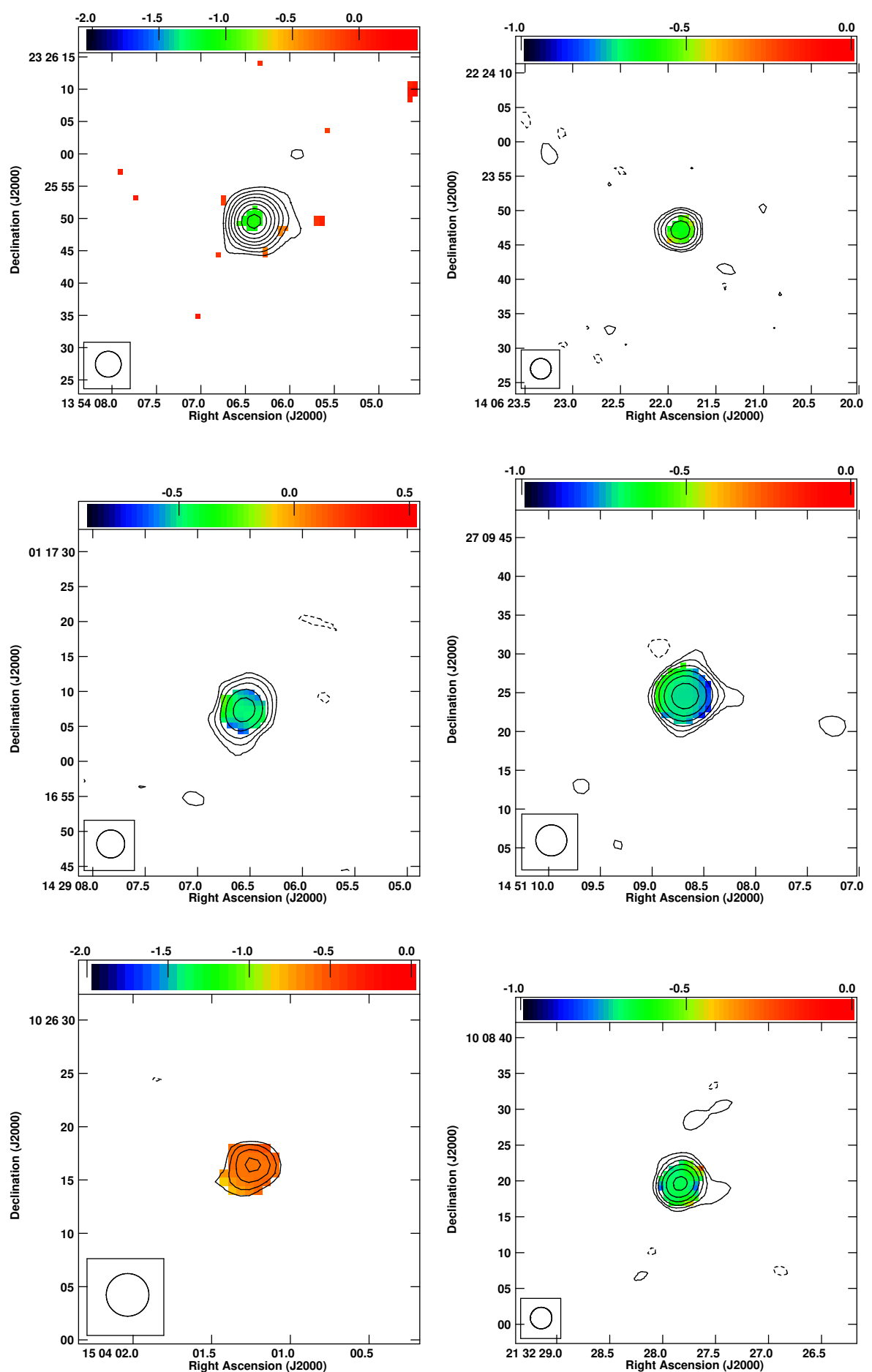

Figure B10. $685 \mathrm{MHz}$ total intensity contours in black superimposed on spectral index image in color for Top left: PG 1351+236; Top right: PG 1404+226; Middle left: PG 1426+015; Middle right: PG 1448+273; Bottom left: PG 1501+106; Bottom right: PG 2130+099. The peak contour flux is $x$ mJy beam $^{-1}$ and the contour levels are $y \times(-1,1,2,4,8,16,32,64,128,256,512) \mathrm{mJy}_{\text {beam }}{ }^{-1}$, where $(x ; y)$ for PG $1404+226$, PG $1426+015$, PG $1448+273$ and PG $2130+099$ are $(132.2 ; 0.10),(51.3 ; 0.09),(17.8 ; 0.10)$ and $(56.9 ; 0.17)$ respectively. The peak contour flux is $x^{*} \mathrm{mJy} \mathrm{beam}^{-1}$ and the contour levels are $y^{*} \times(-1$, $1,1.4,2,2.8,4,5.6,8,11.20,16,23,32,45,64,90,128,180,256,362,512)$ mJy beam $^{-1}$, where $\left(x^{*} ; y^{*}\right)$ for PG $1351+236$ and PG $1501+106$ are $(36.8 ; 0.18)$ and $(1507.6 ; 1.2)$ respectively. 

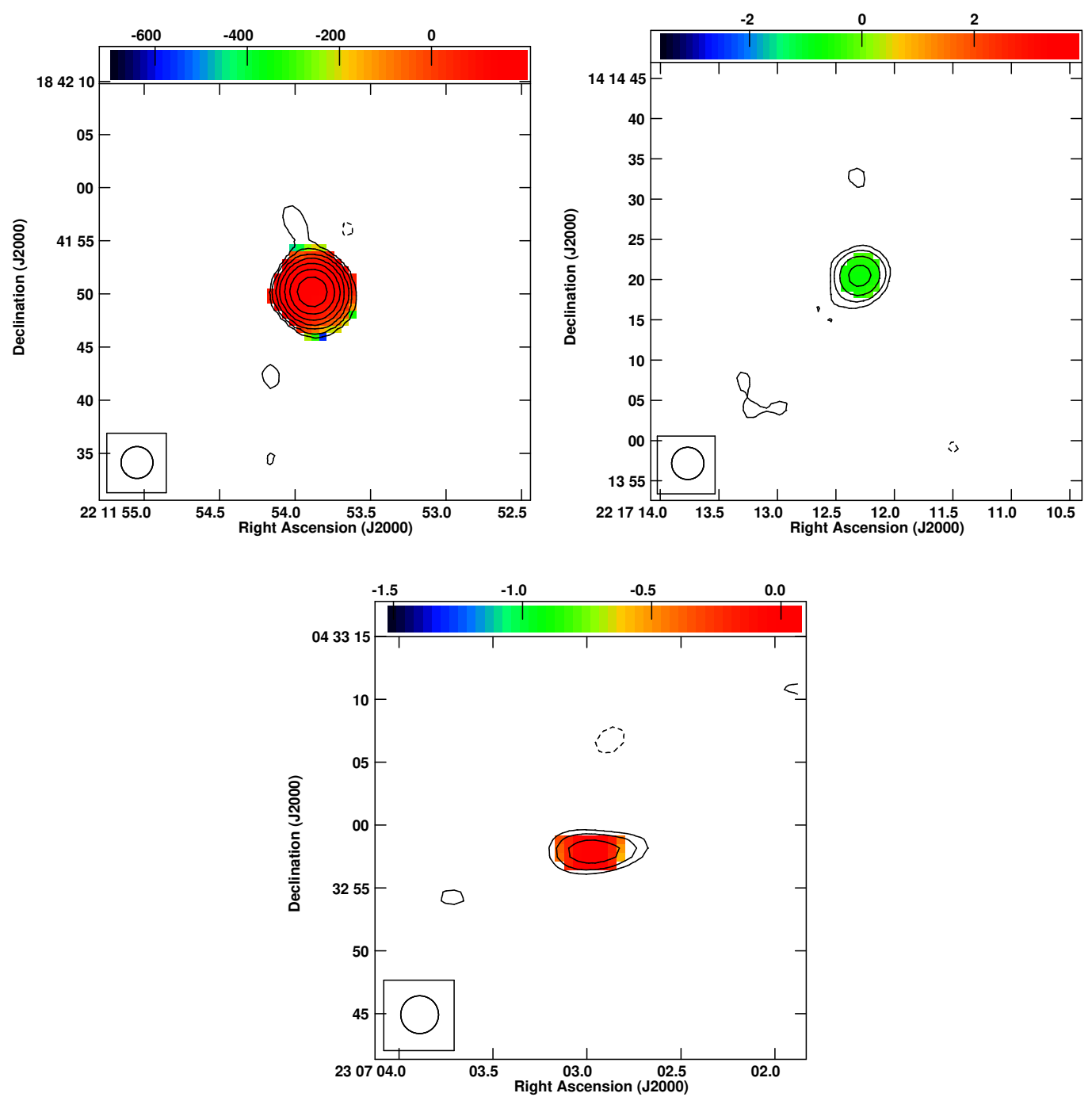

Figure B11. $685 \mathrm{MHz}$ total intensity contours in black superimposed on spectral index image in color for Top left: PG 2209+184; Top right: PG 2214+139; Bottom: PG 2304+042. The peak contour flux is $x \mathrm{mJy}_{\text {beam }}^{-1}$ and the contour levels are $y \times(-1,1,2,4,8,16,32,64,128,256,512) \mathrm{mJy}^{2}$ beam ${ }^{-1}$, where $(x ; y)$ for PG 2209+184 and PG $2214+139$ are $(303 ; 0.80)$ and $(15.60 ; 0.09)$ respectively. The peak contour flux for PG $2304+042$ is $84.5 \mathrm{mJy}^{2}$ beam ${ }^{-1}$ and the contour levels are $0.35 \times(-1,1,1.4,2,2.8,4,5.6,8,11.20,16,23,32,45,64,90,128,180,256,362,512) \mathrm{mJy} \mathrm{beam}^{-1}$. 